Draft version August 16, 2018

Preprint typeset using LATEX style emulateapj v. 11/10/09

\title{
MINOR MERGER-INDUCED COLD FRONTS IN ABELL 2142 AND RXJ1720.1+2638*
}

\author{
Matt S. Owers ${ }^{1}$, Paul E.J. Nulsen ${ }^{2}$, Warrick J. Couch ${ }^{1}$ \\ Draft version August 16, 2018
}

\begin{abstract}
We present evidence for the existence of substructure in the "relaxed appearing" cold front clusters Abell 2142 and RXJ1720.1+2638. The detection of these substructures was made possible by comprehensive multi-object optical spectroscopy obtained with the Hectospec and DEep Imaging MultiObject Spectrograph instruments on the $6.5 \mathrm{~m}$ MMT and 10m Keck II telescope, respectively. These observations produced 956 and 400 spectroscopically confirmed cluster members within a projected radius of $3 \mathrm{Mpc}$ from the centers of Abell 2142 and RXJ1720.1+2638, respectively. The substructure manifests itself as local peaks in the spatial distribution of member galaxies and also as regions of localized velocity substructure. For both Abell 2142 and RXJ1720.1+2638, we identify group-scale substructures which, when considering the morphology of the cold fronts and the time since pericentric passage of a perturber estimated from the cold front radii, could plausibly have perturbed the cluster cores and generated the cold fronts observed in Chandra images. The results presented here are consistent with cold fronts being the result of merger activity and with cold fronts in relaxed appearing clusters being due to minor merger activity.
\end{abstract}

Subject headings: galaxies: clusters: individual (Abell 2142, RXJ1720.1+2638) - X-rays: galaxies: clusters

\section{INTRODUCTION}

One of the triumphs of modern cosmology is the success the hierarchical formation model has had in explaining the observed large scale structure of galaxies. In this model, small-scale perturbations in the matter density distribution at early epochs intensify due to the effects of gravity, collapse and merge together to form the galaxies, groups, and clusters observed at later epochs. Since clusters are the structures to form last, the process of hierarchical formation is expected to reveal itself dramatically in such systems. This is borne out by observations, where many clusters in the local universe are found to have recently undergone significant growth via either a major merger with another cluster or through minor mergers with smaller clusters and groups of galaxies. This offers a unique opportunity to study the effects of large scale structure formation on the constituent galaxies and intra cluster medium (ICM). However, both the detection and distinction of clusters undergoing major or minor mergers can be an observationally expensive exercise in the absence of easily detectable signatures of each process (Owers et al. 2009b).

In this vein, the "cold fronts" first detected and characterized by the Chandra X-ray satellite (Markevitch et al. 2000, Vikhlinin et al. 2001), thanks to its excellent sensitivity and unrivalled spatial resolution, may play an important role. Cold fronts are contact discontinu-

\footnotetext{
${ }^{1}$ Center for Astrophysics and Supercomputing, Swinburne University of Technology, Hawthorn, VIC 3122, Australia; mowers@astro.swin.edu.au

${ }^{2}$ Harvard Smithsonian Center for Astrophysics, 60 Garden Street, Cambridge, MA 02138, USA

* Some of the data presented herein were obtained at the W.M. Keck Observatory, which is operated as a scientific partnership among the California Institute of Technology, the University of California and the National Aeronautics and Space Administration. The Observatory was made possible by the generous financial support of the W.M. Keck Foundation.
}

ities in the ICM which form at abrupt interfaces between low and high entropy plasma (for a review see Markevitch \& Vikhlinin 2007). In the scenario put forth by Markevitch et al. (2000), commonly referred to as the "remnant core" scenario, the edges delineate the low entropy gas of two cool cores, which have just survived a pericentric passage, and the surrounding hot shocked atmospheres which were stripped from the cores during the merger. This remnant core scenario is observed in a number of clusters, e.g., 1ES0657-558 (otherwise known as the Bullet cluster; Markevitch et al. 2002), Abell 1758 (David \& Kempner 2004), Abell 115 (Gutierrez \& Krawczynski 2005), Abell 2744 (Kempner \& David 2004, Owers et al. 2011), Abell 2146 (Russell et al. 2010) and probably Abell 2069 (Owers et al. 2009b). As the number of clusters observed by Chandra increased, two things emerged (i)The remnant core scenario cannot explain the majority of the observed cold fronts, and (ii)-There is a clear dichotomy when considering the X-ray morphology of clusters hosting cold fronts (Markevitch \& Vikhlinin 2007, Owers et al. 2009b, Ghizzardi et al. 2010). One subset of cold front clusters exhibit clearly disturbed X-ray morphologies, while the second subset have cool cores and appear relaxed at large radii. The disturbed morphology present in the former subset is clearly related to major merger activity which manifests itself at other wavelengths. The cold fronts due to the remnant core scenario are generally found in these clusters, but other mergerrelated processes also produce cold fronts (e.g., the "ram pressure slingshot" feature in Abell 168 reported by Hallman \& Markevitch 2004; see also Poole et al. 2006 for different cold fronts produced in merger simulations). In these cases, cold fronts are excellent signatures of major merger activity (Owers et al. 2009a, c). For the subset with relaxed large scale X-ray morphologies, the best evidence for merger activity comes from the observed cold 
fronts - the evidence for merger activity at other wavelengths in these clusters is generally ambiguous or nonexistent.

The leading hypothesis for the formation of cold fronts in clusters with a regular X-ray morphology is the relative motion of the low entropy core gas-termed "sloshing" "4 by Markevitch et al. (2001) - with respect to the quasi-static cluster potential. The simulations of Tittley \& Henriksen (2005), Ascasibar \& Markevitch (2006) and Roediger et al. (2011) showed that a gravitational perturbation in the form of an infalling subcluster without a gaseous atmosphere can induce long-lasting oscillations of the the dark matter and gas cores. While the precise details differ (e.g., in the Tittley \& Henriksen scenario, the dark matter oscillates, dragging the gas with it, while in Ascasibar \& Markevitch (2006) the dark matter is nearly static while the gas oscillates) in all cases the relative motion of low entropy cool core gas with respect to the cluster on larger scales produces cold fronts where it meets the higher entropy gas at larger radii. Hydrodynamical perturbations in the form of minor mergerrelated weak shocks (Churazov et al. 2003), shocks from fast moving galaxies (Roediger et al. 2011), or acousticgravity waves related to bulk motions outside of the cluster cores (Fuiita et al. 2004) have also been investigated by simulations. In these scenarios, the core gas is displaced from the static dark matter core and oscillates around the potential minimum as the system relaxes back to its equilibrium configuration. Several authors have suggested that outbursts from AGN may provide a sufficient perturbation to induce sloshing (Markevitch et al. 2001, Hlavacek-Larrondo et al. 2011) although this possibility is yet to be fully explored. The emerging consensus is that the cold fronts seen in relaxed appearing cluster are due to minor merger activity. This leads to the interesting implication that cold fronts can be used as both a signpost of merger activity and, in combination with X-ray morphology, a tool to differentiate clusters undergoing major and minor mergers.

Much of the focus on sloshing cold front clusters has been at X-ray wavelengths or has involved simulations designed to explore mechanisms which are capable of reproducing the cold fronts along with a relaxed morphology at larger radii. To date, no studies have focused on identifying possible perturbers by utilizing the spatial and kinematic information provided by multiobject spectroscopy (MOS) - an issue critical to the minor merger scenario where a recently merged perturber is not apparent at X-ray wavelengths, but may manifest itself as a dynamical substructure. Here, we set out to address this problem using the powerful combination of spatial and redshift information provided by multiobject spectroscopy in an attempt to detect merger related substructure and characterize its dynamical properties. Such observations are a crucial test for constraining simulations and for showing that cold fronts are excellent probes of ongoing merger activity. In this paper we present and analyze comprehensive MOS observations of the sloshing cold front clusters Abell 2142 and RXJ1720.1+2638 (hereafter A2142 and RXJ1720,

\footnotetext{
${ }^{4}$ For clarity, throughout the paper we will refer to clusters which have regular/relaxed X-ray morphologies with cold fronts in their cores as sloshing cold front clusters.
}

respectively) which were selected from the subsample of relaxed appearing cold front clusters in Owers et al. (2009b). Previous MOS follow up of cold front clusters taken from the disturbed subset in Owers et al. (2009b) has demonstrated that MOS observations are an essential ingredient required for understanding the merging history of a cluster (Owers et al. 2009c, a).

A2142 was one of the first clusters targeted with the Chandra X-ray telescope and, along with the observations of Abell 3667 (Vikhlinin et al. 2001), characterized for the first time the cold front phenomenon (Markevitch et al. 2000). The Einstein X-ray images of A2142 presented in Oegerle et al. (1995), revealed an asymmetric X-ray morphology, while their analysis of 103 spectroscopically confirmed members of this cluster yielded a mean redshift of $z=0.0905 \pm$ 0.0004 and a velocity dispersion $\sigma=1280_{-76}^{+94} \mathrm{~km} \mathrm{~s}^{-1}$. Oegerle et al. (1995) performed a Dressler-Schectman test (Dressler \& Shectman 1988) and found marginal evidence for dynamical substructure, while their data also revealed a high peculiar velocity for the second ranked member of $\sim 1600 \mathrm{kms}^{-1}$. X-ray observations using ROSAT revealed a highly elliptical X-ray morphology with a luminous compact core, the centroid of which is offset from that of the larger scale emission (Buote \& Tsai 1996, Henry \& Briel 1996). The temperature map of Henry \& Briel (1996) showed that this compact core was cooler than the surrounding gas and that the temperature distribution was asymmetric on large scales. The high ellipticity, offset core, asymmetric temperature distribution and evidence for dynamical structure were interpreted by both Henry \& Briel and Buote \& Tsai as evidence for a later stage merger (i.e., viewed $\sim 1$ Gyr after the merger) in A2142. Hints of a substructure to the northwest of the core in A2142 are seen in the weak lensing and SZ maps presented in Umetsu et al. (2009), and also the lensing data of Okabe \& Umetsu (2008).

RXJ1720 was categorized as an extended X-ray source in the Einstein Slew Survey (Elvis et al. 1992). The Chandra observations of RXJ1720 revealed two cold fronts within $100^{\prime \prime}$ of the cluster center, while the X-ray emission outside of this radius showed a regular morphology (Mazzotta et al. 2001). These Chandra observations of RXJ1720 provided the first clear indication that cold fronts can also be found in relaxed appearing cool core clusters. Mazzotta et al. (2001) interpreted the cold fronts as evidence for either a late stage major merger viewed after several pericentric passages or the asynchronous collapse of two nearly cospatial density perturbations with different linear scales. Using a deeper Chandra observation, Mazzotta \& Giacintucci (2008) found that the spiral features revealed in the temperature and residual maps are more readily explained by the sloshing scenario. Mazzotta \& Giacintucci also reported an interesting correlation between the spiral structure and radio emission from a mini-halo and suggested that the origin of this diffuse radio emission may be related to the sloshing process. At optical wavelengths, RXJ1720 was detected with the C4 cluster finding algorithm using the Sloan Digital Sky Survey (SDSS) (Miller et al. 2005). From 26 spectroscopically confirmed cluster members Miller et al. (2005) measured a mean redshift of 
$z=0.1603$ and a velocity dispersion $\sigma=878 \mathrm{~km} \mathrm{~s}^{-1}$ while the Dressler-Schectman test revealed no evidence for dynamical substructure. The projected mass maps from the weak lensing analysis of Okabe et al. (2010) showed that RXJ1720 harbors a second mass condensation just north of the main cluster component which is indicative of merger activity.

For both clusters there are tantalizing hints for merger activity. The goal of this paper is to seek conclusive evidence of dynamical substructure related to merger activity by using the extensive spectroscopy collected at the $6.5 \mathrm{~m}$ MMT and $10 \mathrm{~m}$ Keck II Telescope using the Hectospec and DEep Imaging Multi-Object Spectrograph instruments, respectively. We describe our target selection and MOS observations in Section 2 and our methods for cluster member selection and substructure detection in Section 3. We interpret our results in Section 4 and present a summary and concluding remarks in Section 5 Throughout this paper we assume a standard $\Lambda \mathrm{CDM}$ cosmology where $H_{0}=70 \mathrm{~km} \mathrm{~s}^{-1} \mathrm{Mpc}^{-1}, \Omega_{m}=0.3$ and $\Omega_{V}=0.7$. Assuming this cosmology, at the systemic redshifts of A2142 and RXJ1720, 1 arcsecond corresponds to a physical scale of $1.68 \mathrm{kpc}$ and $2.76 \mathrm{kpc}$, respectively.

\section{OBSERVATIONS AND DATA REDUCTION}

\subsection{Photometric Catalogs}

The spectroscopic target catalogs for both A2142 and RXJ1720 were drawn from the SDSS and included objects within a projected distance from the cluster center of $R_{\text {proj }}=3 \mathrm{Mpc}$, or $\sim 30^{\prime}$ and $\sim 18^{\prime}$ for $\mathrm{A} 2142$ and RXJ1720, respectively. The catalogs were filtered to contain only objects identified as galaxies by the SDSS pipeline morphological classification scheme (Stoughton et al. 2002). Objects near to bright stars and misclassified as galaxies by the automated SDSS pipeline were identified by eye and rejected from the catalog. Objects with existing SDSS spectroscopy and having redshifts which placed them well outside the redshift range expected for the cluster of interest were also removed from the sample. We transformed the SDSS $g$ and $r$-band magnitudes onto the Johnson-Cousins $B$ and $R$ system using Equations $\mathrm{A} 5$ and $\mathrm{A} 7$ of Cross et al. (2004).

\subsection{MMT/Hectospec Observations}

The majority of the spectra used in this paper were collected at the $6.5 \mathrm{~m}$ MMT using the Hectospec multiobject spectrograph (Fabricant et al. 2005). Hectospec is a bench-mounted spectrograph which has the capability to simultaneously observe 300 objects through $1^{\prime \prime} .5$ diameter fibers spread over a 1 degree field of view. The observations were performed with the 270 groove $\mathrm{mm}^{-1}$ grating resulting in spectra with $\sim 6 \AA$ resolution covering the wavelength range $3500-10000 \AA$. Approximately 30 fibers were allocated to blank sky regions to facilitate sky subtraction during the data reduction. Due to the large number of targets compared to the number of available fibers and fiber packing limitations, 6 and 8 configurations were required to obtain adequate spectroscopic completeness for RXJ1720 and A2142, respectively. The exposure time, magnitude limit and seeing conditions for each MMT/Hectospec configuration are summarized in Table 1.
The fiber configurations were generated with the XFITFIBS5 software where the ranking capability was utilized to ensure that the most likely cluster members had a better chance of being allocated to a configuration. For RXJ1720, objects were ranked based on their position on the color-magnitude relation (Figure 1) and also their $R_{\text {proj }}$ in the sense that objects lying redward of the cluster red-sequence, i.e., above the bold black line in Figure 1 and with $2.5<R_{\text {proj }}<3 \mathrm{Mpc}$ were ranked lowest while those galaxies which lie on or blueward of the red-sequence and with $R_{\text {proj }}<0.5 \mathrm{Mpc}$ were ranked highest. The slope of the line delineating those galaxies redward of the red sequence is calculated from the best fit to the red sequence slope versus redshift diagram in Figure 3. of López-Cruz et al. (2004). The ranking procedure was similar for the A2142 configurations, except that objects lying redward of the cluster red-sequence were simply not included for observation (Figure 11), while a number of objects identified as being part of local projected overdensities (aside from the central overdensity) were given higher rankings and a number of fainter $(20<R \leq 20.5)$ objects were included with low rankings as filler objects to be allocated to spare fibers.

The spectra were reduced at the Smithsonian Astrophysical Observatory Telescope Data Center[ (TDC) using the SPECROAD pipeline7 (Mink et al. 2007). Redshifts were also determined at the TDC using the IRAF cross-correlation XCSAO software (Kurtz et al. 1992) and each spectrum was assigned a redshift quality of "Q" for a reliable redshift, "?" for questionable and "X" for a bad redshift measurement. These observations provided 1771 and 815 quality "Q" redshift measurements for extragalactic objects in the A2142 and RXJ1720 fields, respectively.

\subsection{Keck/DEIMOS Observations}

The MMT/Hectospec data for RXJ1720 were supplemented with spectra obtained at the Keck II telescope using the Deep Imaging Multi-Object Spectrograph (DEIMOS). In MOS mode, DEIMOS utilizes a slitmask to collect spectra for $\sim 100$ target objects positioned within $\mathrm{a} \sim 16.7^{\prime} \times 5^{\prime}$ field of view. The observations presented here made use of the 600 line $\mathrm{mm}^{-1}$ grating which, in combination with a $1^{\prime \prime} .5$ slitwidth, gives a spectral resolution of $\sim 7.0 \AA$. The GG400 order-blocking filter was used and the grating was tilted to a central wavelength of $5500 \AA$ which yields a useable spectral range of $\sim 4000-9000 \AA$.

Slit masks were generated with the DSIMULATOR task within the DEIMOS IRAF package. The input target catalog was limited to contain objects with $R<22$ and within $R_{\text {proj }}=2 \mathrm{Mpc}\left(\sim 12^{\prime}\right)$. The catalog was culled of those objects found to be non-cluster members from the previous MMT/Hectospec observations, while those spectroscopically confirmed cluster members with high quality Hectospec spectra (i.e. $\mathrm{S} / \mathrm{N} \gtrsim 8$ ) were also removed. The DSIMULATOR software allows a two tier ranking of targets: First, the catalog is split into subsamples with objects in the first subsample given first priority

\footnotetext{
${ }^{5}$ http://www.cfa.harvard.edu/mmti/hectospec.html

6 http://tdc-www.harvard.edu

${ }^{7}$ http://tdc-www.harvard.edu/instruments/hectospec/specroad.html
} 

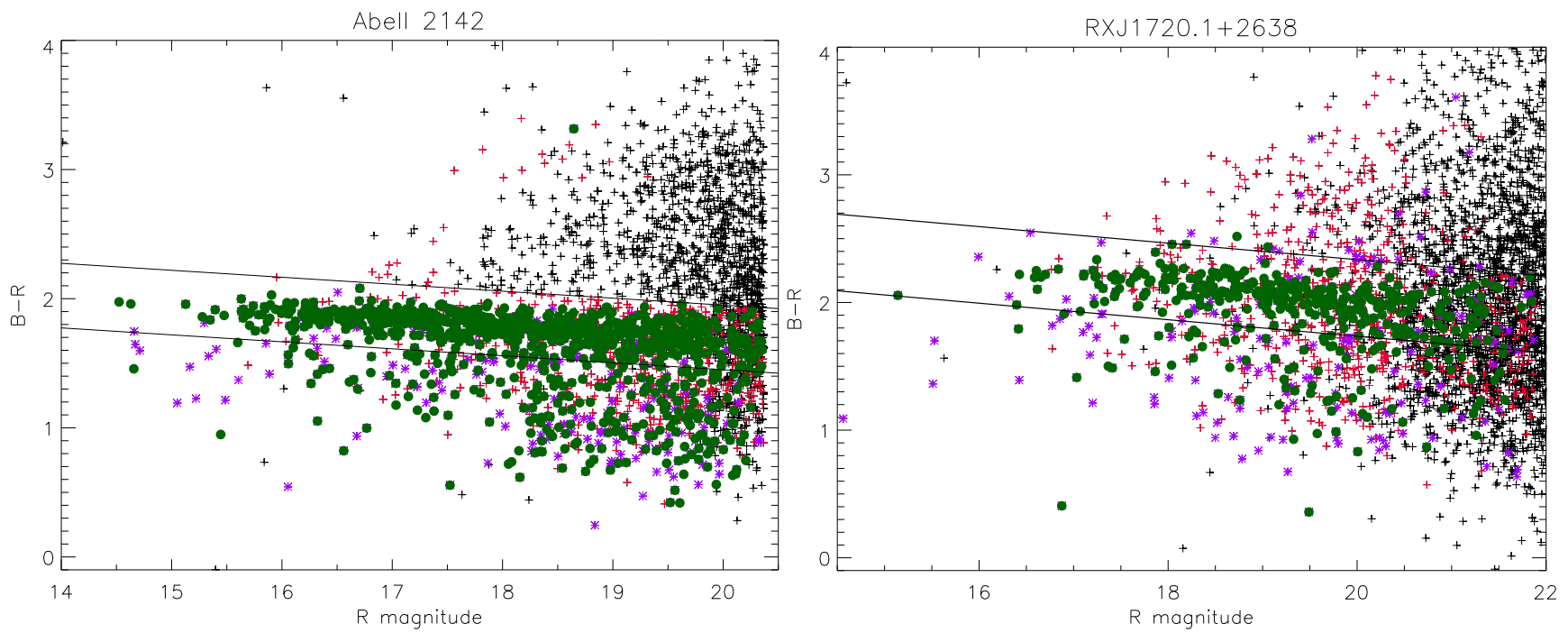

Figure 1. Color magnitude diagrams for the objects within $3 \mathrm{Mpc}$ of A2142 (left panel) and RXJ1720 (right panel). Filled green circles are spectroscopically confirmed cluster members, blue stars are foreground objects, red crosses are background objects and black crosses are objects without a spectrum. The black lines show the estimates used for the red and blue limits defining the cluster red envelope which was used for the cluster membership allocation procedure described in Section 3.1 The red limit was used in the ranking process described in Sections 2.2 and 2.3

Table 1

Summary of the MMT/Hectospec observations.

\begin{tabular}{ccccccc}
\hline \hline Cluster & Date & Magnitude limit & Exposure Time (s) & $\mathrm{N}_{\mathrm{spec}}$ & $\mathrm{N}_{\mathrm{z}}$ & Seeing \\
\hline RXJ1720 & 2006 May 4 & $R<20.5$ & $3 \times 900 \mathrm{~s}$ & 208 & 189 & - \\
& 2006 May 4 & $R<20.5$ & $3 \times 900 \mathrm{~s}$ & 186 & 176 & - \\
& 2007 Mar 15 & $R<20.5$ & $3 \times 900 \mathrm{~s}$ & 189 & 171 & $0^{\prime \prime} .97$ \\
& 2007 Mar 16 & $R<20.5$ & $3 \times 1200 \mathrm{~s}$ & 177 & 92 & $0^{\prime \prime} .63$ \\
& 2007 April 16 & $R<20.5$ & $3 \times 1200 \mathrm{~s}$ & 161 & 122 & $1^{\prime \prime} .5$ \\
& 2007 April 20 & $R<20.5$ & $5 \times 600 \mathrm{~s}$ & 166 & 121 & $1^{\prime \prime} .3$ \\
$\mathrm{~A} 2142$ & 2009 February 5 & $R<20.5$ & $3 \times 1200 \mathrm{~s}$ & 266 & 255 & $1^{\prime \prime} .08$ \\
& 2009 February 21 & $R<20.5$ & $3 \times 1200 \mathrm{~s}$ & 261 & 174 & $1^{\prime \prime} .30$ \\
& 2009 February 24 & $R<20.5$ & $1 \times 1200 \mathrm{~s}$ & 263 & 159 & $0^{\prime \prime} .56$ \\
& 2009 March 1 & $R<20.5$ & $3 \times 1200 \mathrm{~s}$ & 263 & 249 & $0^{\prime \prime} .84$ \\
& 2009 April 22 & $R<20.5$ & $3 \times 960 \mathrm{~s}$ & 260 & 255 & $0^{\prime \prime} .60$ \\
& 2009 April 23 & $R<20.5$ & $3 \times 1200 \mathrm{~s}$ & 259 & 244 & $1^{\prime \prime} .21$ \\
& 2009 April 23 & $R<20.5$ & $3 \times 1200 \mathrm{~s}$ & 255 & 240 & $0^{\prime \prime} .61$ \\
& 2009 April 24 & $R<20.5$ & $3 \times 1200 \mathrm{~s}$ & 242 & 229 & $0^{\prime \prime} .86$
\end{tabular}

in slit allocation. Second, the objects within subsamples are given weights such that the highest weighted objects are given a higher priority during slit allocation. We divide the catalog into 5 subsamples: the first and second contain objects lying on or blueward of the red sequence with $R \leq 21.5$ and $21.5<R \leq 22$, respectively, the third contains objects already observed with Hectospec, the fourth and fifth contain objects lying redward of the red sequence with $R \leq 21.5$ and $21.5<R \leq 22$, respectively. The object weights were assigned according to $R_{\text {proj. }}$. This subsampling and weighting scheme means that objects within the central $500 \mathrm{kpc}$ lying on or blueward of the red sequence and with $R \leq 21.5$ are most likely to be allocated a slit, while objects with $21.5<R \leq 22$ lying redward of the red sequence and $2 \mathrm{Mpc}$ from the cluster center are least likely to be allocated a slit on the mask. The position angle of each slit may be tilted by \pm 30 deg with respect to the mask position angle (which is aligned with the spatial direction on the CCD). Where the position angle of a galaxy's major axis (taken from the SDSS shape parameters) does not exceed this limi- tation, we tilt the slit to align with the major axis. In practice, this was only possible in a few cases and the majority of slits were therefore assigned a position angle of $5 \mathrm{deg}$ with respect to the mask. Using this setup, we generated 6 slitmasks which were observed on 2009 May 24. Table 2 summarizes these observations.

The data were reduced with the spec $2 \mathrm{~d}$ software 8 developed for the DEEP2 galaxy redshift survey (Davis et al. 2003). Briefly9, the software uses the internal Quartz flats taken on the afternoon before the observations to find and rectify the slits on the CCD, to determine the throughput of each slit and to correct for fringing. We utilize blue and red specific arc lamps to determine the 2D wavelength solution which is initially estimated using the DEIMOS optical model. The science exposures are combined using an inverse variance weighed mean after cosmic ray correction and sky subtraction. The $1 \mathrm{D}$ spectra are then extracted using optimal extraction (Horne

8 http://deep.berkeley.edu/spec2d/

9 a more thorough explanation can be found at http://deep.berkeley.edu/spec2d/primer.html 
Table 2

Summary of the Keck/DEIMOS observations of RXJ1720.

\begin{tabular}{ccccccc}
\hline \hline$\alpha(\mathrm{J} 2000)$ & $\delta(\mathrm{J} 2000)$ & Mask PA & Exposure Time $(\mathrm{s})$ & $\mathrm{N}_{\mathrm{spec}}$ & $\mathrm{N}_{\mathrm{z}}$ & Seeing \\
\hline $17: 20: 05.02$ & $26: 39: 41.5$ & 180.0 & $2 \times 1100+1340 \mathrm{~s}$ & 70 & 61 & $0^{\prime \prime} .57$ \\
$17: 20: 14.28$ & $26: 35: 38.2$ & 0.0 & $2 \times 1100+1340 \mathrm{~s}$ & 67 & 67 & $0^{\prime \prime} .60$ \\
$17: 20: 03.70$ & $26: 37: 31.9$ & 0.0 & $2 \times 1100+1200 \mathrm{~s}$ & 62 & 57 & $0^{\prime \prime} .50$ \\
$17: 20: 16.73$ & $26: 37: 25.3$ & 180.0 & $2 \times 1100+1200 \mathrm{~s}$ & 60 & 57 & $0^{\prime \prime} .63$ \\
$17: 19: 46.02$ & $26: 37: 31.9$ & 0.0 & $2 \times 1100+1500 \mathrm{~s}$ & 68 & 68 & $0^{\prime \prime} .54$ \\
$17: 20: 34.44$ & $26: 37: 31.9$ & 180.0 & $2 \times 1100+800 \mathrm{~s}$ & 62 & 50 & $0^{\prime \prime} .61$
\end{tabular}

1986) and rebinned to linear wavelength bins using the IRAF task RSPECTEXT.

The redshifts are again measured with the XCSAO cross-correlation package in IRAF (Kurtz et al. 1992) and each spectrum was visually assessed (by MSO) and assigned a redshift quality of "Q" for a reliable redshift, "?" for questionable and "X" for a bad redshift measurement. These observations produced 333 quality "Q" redshift measurements for extragalactic objects in the RXJ1720 field.

\subsection{Redshift precision}

The redshift precision is determined here using repeat observations taken during the observating runs. For the MMT/Hectospec observations of A2142, there were 234 objects having two quality "Q" redshift determinations which were reobserved due to their lower signal to noise spectra (none were taken for RXJ1720). Taking the difference of the redshifts of these repeat observations (in the sense that we subtract the subsequent higher $\mathrm{S} / \mathrm{N}$ redshift measurement from the lower $\mathrm{S} / \mathrm{N}$ initial redshift) we use biweight estimators to find a mean redshift difference of $\langle\Delta c z\rangle=2.3 \pm 2.3 \mathrm{kms}^{-1}$ and a standard deviation $\sigma(\Delta c z)=34.2 \pm 2.3 \mathrm{~km} \mathrm{~s}^{-1}$ indicating a single measurement uncertainty of $\sigma(<\Delta c z>) / \sqrt{2}=$ $24.2 \pm 1.6 \mathrm{~km} \mathrm{~s}^{-1}$. This single measurement uncertainty is consistent with the median of the redshift uncertainties determined by the XCSAO software of $24.7 \mathrm{~km} \mathrm{~s}^{-1}$.

We can test the veracity of this internal precision measurement by comparing the repeat observations taken with MMT/Hectospec and from the SDSS. There are 301 objects which have redshift measurements from both our A2142 MMT/Hectospec observations and the SDSS. Taking the difference between the MMT/Hectospec and SDSS redshifts, we find $\langle\Delta c z\rangle=-4.8 \pm 1.8 \mathrm{~km} \mathrm{~s}^{-1}$ and $\sigma(\Delta c z)=31.3 \pm 1.5 \mathrm{~km} \mathrm{~s}^{-1}$. This standard deviation is well encompassed by the median of the individual redshift uncertainty measurements for the SDSS $\left(\overline{c z_{\mathrm{err}}}=47.5 \mathrm{~km} \mathrm{~s}^{-1}\right)$ and the MMT $/$ Hectospec $\left(\overline{c z_{\mathrm{err}}}=\right.$ $\left.21.9 \mathrm{~km} \mathrm{~s}^{-1}\right)$ observations. Similarly, while there were no repeat observations within the MMT/Hectospec or Keck/DEIMOS redshift catalogues for RXJ1720, there were 52 common measurements between the SDSS and MMT/Hectospec. The mean difference in the redshift measurements common to the SDSS and Hectospec catalogues was $\langle\Delta c z\rangle=-31.6 \pm 7.4 \mathrm{~km} \mathrm{~s}^{-1}$ and $\sigma(\Delta c z)=$ $49.8 \pm 6.3 \mathrm{kms}^{-1}$. The quadrature sum of the median value for the individual uncertainties on the SDSS $\left(\overline{c z_{\mathrm{err}}}=45.8 \mathrm{~km} \mathrm{~s}^{-1}\right)$ and the MMT/Hectospec $\left(\overline{c z_{\mathrm{err}}}=\right.$ $\left.28.9 \mathrm{~km} \mathrm{~s}^{-1}\right)$ repeat observations well encompasses the scatter in $\langle\Delta c z\rangle$ implying that the individual uncer- tainties provide a good measure of the real uncertainties associated with these measurements.

Given the robustness of the MMT/Hectospec redshift measurements and associated uncertainties, we now use the $33 \mathrm{MMT} /$ Hectospec objects which were reobserved with Keck/DEIMOS to determine the real uncertainties in the DEIMOS measurements, finding $\langle\Delta c z\rangle=$ $-69.5 \pm 19.0 \mathrm{~km} \mathrm{~s}^{-1}$ with scatter $\sigma(\Delta c z)=111.6 \pm$ $18.5 \mathrm{~km} \mathrm{~s}^{-1}$. Comparing the median values of the individual uncertainty measurements (for MMT/Hectospec: $\overline{c z_{\mathrm{err}}}=38.5 \mathrm{~km} \mathrm{~s}^{-1}$ and for Keck/DEIMOS: $\overline{c z_{\mathrm{err}}}=$ $52.3 \mathrm{~km} \mathrm{~s}^{-1}$ ) it can be seen that the scatter is not well accounted for by the quadrature sum of the uncertainties. We conclude that the real redshift uncertainties on the DEIMOS measurements are $\sim 100 \mathrm{~km} \mathrm{~s}^{-1}$.

Since there are significant offsets measured in the repeat observations for RXJ1720, when combining the MMT/Hectospec, Keck/DEIMOS and SDSS catalogs we correct each Keck/DEIMOS and SDSS redshift measurement by the $\langle\Delta c z\rangle$ value measured above such that they are consistent with the MMT/Hectospec measurements. Where there are repeat measurements, for both the A2142 and RXJ1720 catalogs we use the measurement with the lowest measured uncertainty. Where a Keck/DEIMOS measurement is involved, we double the redshift uncertainty, consistent with the results outlined above. The final catalogs contain 1121 and 1635 single redshift measurements for extragalactic objects in the field surrounding RXJ1720 and A2142, respectively. We tabulate the position, redshift, redshift uncertainty and redshift source of objects in the final catalogs in Tables 3 and 4 for A2142 and RXJ1720, respectively.

\subsection{Spectroscopic completeness}

An understanding the spectroscopic completeness of our redshift catalogs is imperative when interpreting the results of tests for substructure, in particular for those that rely on finding local overdensities in the spatial distribution of the cluster members. More specifically, we need to understand what fraction of potential cluster members are in the photometric catalog but have not been assigned a redshift. Therefore, we define the spectroscopic completeness as the ratio of the number of objects with quality "Q" redshift measurements to the number of objects in the photometric catalog. As seen in the right panel of Figure 1. objects lying redward of the cluster red sequence are unlikely to be cluster members. Therefore, given our goal of understanding how redshift incompleteness affects the cluster members, for RXJ1720 we only measure the spectroscopic completeness for the subsample of objects lying below the upper black bold line defining the red limit of the cluster red sequence in 
Table 3

Combined redshift catalog for A2142.

\begin{tabular}{ccccc}
\hline \hline $\begin{array}{c}\text { R.A. (J2000) } \\
\text { deg. }\end{array}$ & $\begin{array}{c}\text { decl. (J2000) } \\
\text { deg. }\end{array}$ & $\begin{array}{c}(c z) \\
\mathrm{km} \mathrm{s}^{-1}\end{array}$ & $\begin{array}{c}c z \text { uncertainty } \\
\mathrm{km} \mathrm{s}^{-1}\end{array}$ & $c z$ source \\
\hline 239.036453 & 27.235901 & 57055.78 & 18.59 & MMT/Hectospec \\
239.042587 & 27.177689 & 28939.35 & 30.64 & MMT/Hectospec \\
239.045349 & 27.174765 & 28811.72 & 56.53 & MMT/Hectospec \\
239.048401 & 27.247974 & 96294.95 & 58.26 & MMT/Hectospec \\
239.049500 & 27.187420 & 24531.00 & 48.49 & MMT/Hectospec \\
239.053802 & 27.157728 & 86498.82 & 35.92 & MMT/Hectospec \\
239.055679 & 27.257549 & 33359.37 & 35.87 & MMT/Hectospec \\
239.062576 & 27.132755 & 97387.20 & 21.20 & MMT/Hectospec \\
239.078674 & 27.185207 & 67723.70 & 27.55 & MMT/Hectospec \\
239.079071 & 27.307705 & 26771.70 & 19.48 & MMT/Hectospec \\
& & & & \\
\hline
\end{tabular}

Note. - This table is available in its entirety in a machine-readable form in the online journal. A portion is shown here for guidance regarding its form and content.

Table 4

Combined redshift catalog for RXJ1720.

\begin{tabular}{ccccc}
\hline \hline $\begin{array}{c}\text { R.A. (J2000) } \\
\text { deg. }\end{array}$ & $\begin{array}{c}\text { decl. (J2000) } \\
\text { deg. }\end{array}$ & $\begin{array}{c}(c z) \\
\mathrm{km} \mathrm{s}^{-1}\end{array}$ & $\begin{array}{c}c z \text { uncertainty } \\
\mathrm{km} \mathrm{s}^{-1}\end{array}$ & $c z$ source \\
\hline 259.713308 & 26.620569 & 119987.97 & 102.64 & MMT/Hectospec \\
259.718571 & 26.644934 & 47540.42 & 144.19 & MMT/Hectospec \\
259.719717 & 26.702864 & 111920.71 & 101.03 & MMT/Hectospec \\
259.726667 & 26.565548 & 72713.53 & 28.23 & MMT/Hectospec \\
259.727325 & 26.544941 & 97930.56 & 28.17 & MMT/Hectospec \\
259.728700 & 26.560825 & 72656.78 & 80.44 & MMT/Hectospec \\
259.731187 & 26.554123 & 98086.70 & 55.13 & MMT/Hectospec \\
259.732817 & 26.646864 & 36395.72 & 35.47 & MMT/Hectospec \\
259.738025 & 26.516831 & 30848.81 & 21.77 & MMT/Hectospec \\
259.739112 & 26.738709 & 117214.30 & 35.72 & MMT/Hectospec \\
& & & &
\end{tabular}

Note. - This table is available in its entirety in a machine-readable form in the online journal. A portion is shown here for guidance regarding its form and content.

the right panel of Figure 1]. This also allows a more meaningful comparison to the A2142 data. Figure2 shows the spectroscopic completeness as a function of $R_{\text {proj }}$ for both clusters for different magnitude bins. For A2142, it can be seen in the left panel of Figure 2 that for the high priority targets (i.e. those with $R \leq 20$.) the spectroscopic completeness is always higher than $80 \%$, while it drops to $30-40 \%$ for the low priority faint objects. We therefore achieve a high level of spectroscopic completeness to $\sim 3$ magnitudes fainter than the characteristic magnitude of the cluster luminosity function, $M^{*}$.

With regard to RXJ1720, the right panel in Figure 2 shows that we achieve spectroscopic completeness above $\sim 85 \%$ for magnitudes brighter than $R=20.5$ (apart from magnitudes $19.5<R \leq 20.5$ at radii outside $2 \mathrm{Mpc}$ ). At fainter magnitudes than $R=20.5$ our data are limited to the central $\sim 2 \mathrm{Mpc}$ region covered by the Keck/DEIMOS spectroscopy. For these observations, the smaller FOV and more limited number of objects observed per pointing (compared to the Hectospec observations) meant that it was difficult to obtain multiple pointings of the same regions over the entire central area within $2 \mathrm{Mpc}$ of the cluster center. Therefore, the spectroscopic completeness at these faint magnitudes is limited, but still respectable, at $>50 \%$ for radii $<1.5 \mathrm{Mpc}$ and $20.5<R \leq 21.5$. As expected, the low priority $21.5<R \leq 22.0$ objects have low spectroscopic completeness $(\sim 40 \%$ for radii $<1.5 \mathrm{Mpc})$. Given the more heterogeneous coverage for RXJ1720, we further explore the spatial distribution of the spectroscopic completeness. Briefly, we use the WVT binning algorithm by Diehl \& Statler (2006), which is a generalization of Cappellari \& Copin's (2003) Voronoi binning algorithm, to produce an adaptively binned image of the spatial distribution of objects in the photometric subsample such that each bin contains at least 20 galaxies. This binning is then applied to the spatial distribution of objects in the spectroscopic sample. The resulting image is divided by the binned image of the spatial distribution of objects in the photometric sample to produce the completeness map. These maps are produced at three limiting magnitudes of interest: $R \leq 20.5, R \leq 21.5$ and $R \leq 22.0$ and are shown in Figure 3 . The leftmost map in Figure 3 shows the completeness for the magnitude limit of the MMT/Hectospec observations and confirms that the spectroscopic completeness is excellent and generally above $80 \%$. The middle and right panels reveal a more patchy spectroscopic completeness which is high in the central $1 \mathrm{Mpc}$ and falls off towards the cluster outskirts.

\section{ANALYSIS}

\subsection{Allocation of cluster members}

We allocate cluster membership using a two-step procedure. In the first step, crude cluster membership is achieved by selecting galaxies within $3 \mathrm{Mpc}$ of the brightest cluster galaxy (BCG) and with a peculiar velocity of 

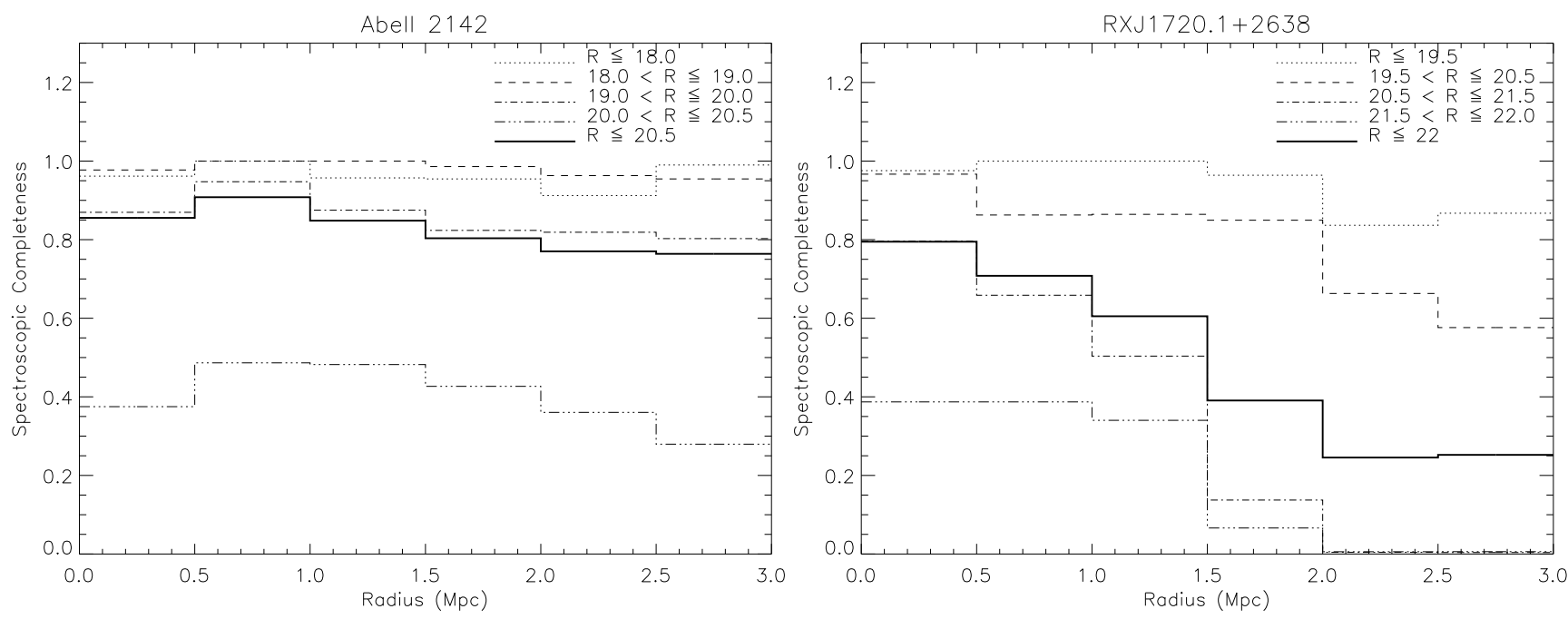

Figure 2. Spectroscopic completeness as a function of radius in different magnitude bins (listed in the top right corner) for A2142 (left panel) and RXJ1720 (right panel).
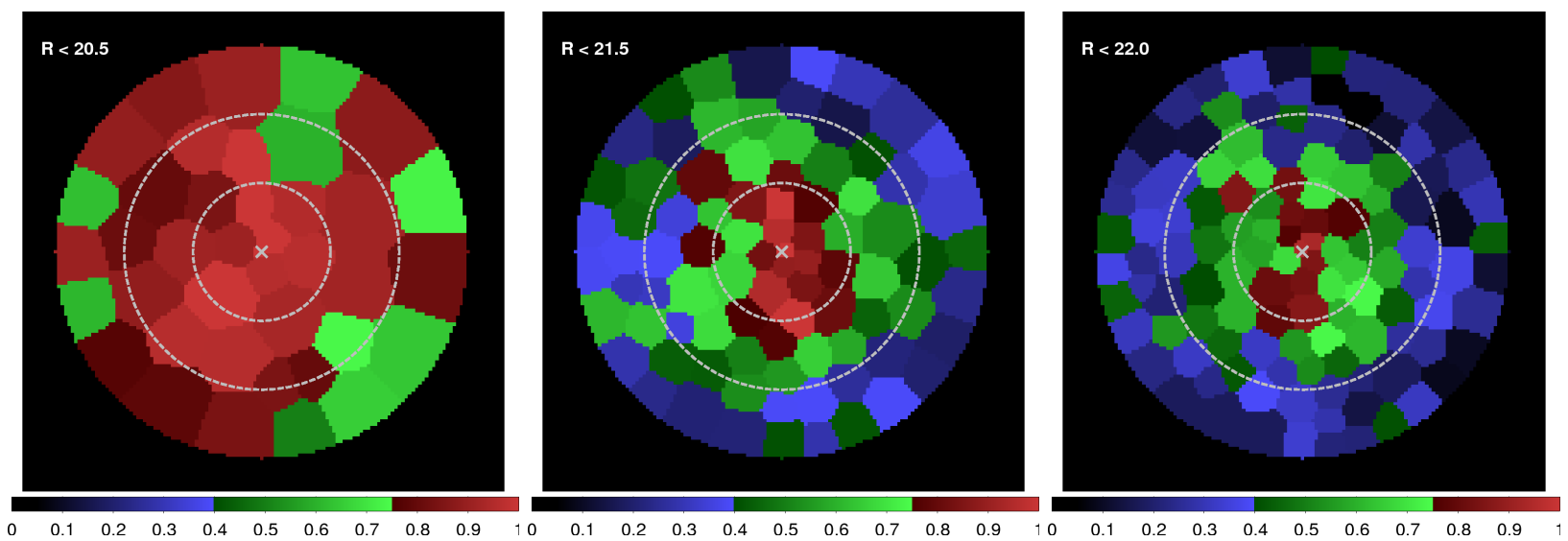

Figure 3. Spectroscopic completeness maps for RXJ1720 for objects with magnitudes $R \leq 20.5$ (left panel), $R \leq 21.5$ (middle panel) and $R \leq 22$ (right panel). The dashed circles have radii of 1 and $2 \mathrm{Mpc}$ and the cross marks the position of the central BCG.

$\pm 10000 \mathrm{~km} \mathrm{~s}^{-1}$ with respect to the BCG redshift. The shifting gapper method used previously in Owers et al. (2009a, c) does not adequately remove the remaining interlopers. This is because the depth and completeness of the A2142 redshift catalog means gaps in velocity phase space are more likely to be "filled", while the proximity of a nearby structure in velocity space for RXJ1720 causes the shifting gapper method to fail (Figure 4). Therefore, we employ the parametric technique introduced by den Hartog \& Katgert (1996) where the maximum possible velocity at the projected radius of each galaxy is calculated given the mass profile of the cluster, which is derived from the virial theorem (for a detailed discussion see Wojtak \& Łokas 2007, Wojtak et al.|2007). Determination of the virial mass relies critically on the assumption that the particles tracing the underlying gravitational potential are virialized and that their distribution is spherically symmetric. The most likely galaxies for which these assumptions apply are elliptical and S0 type galaxies, while spiral galaxies are more likely to be either line of sight interlopers, or an infalling population on radial orbits and, therefore, not virialized (Biviano et al. 1997, Biviano \& Katgert 2004). For these reasons, we use only galaxies on the red sequence shown in Figure 1. which we assume is primarily composed of elliptical and S0 galaxies, to determine the mass profiles used in defining the velocity limits for cluster membership. Furthermore, to reduce any biases in the measurements of the mean projected separations used for the virial mass estimator, we restrict the red sequence galaxies to a magnitude range with high spectroscopic completeness (i.e., $R<19.5$ for RXJ1720 and $R<19.0$ for A2142). The results of the membership allocation are presented in Figure 4 where it can be seen that this method of interloper removal does an excellent job. For reference, we highlight the galaxies lying on the blue cloud using blue stars in Figure 4. We define blue cloud galaxies as those lying blueward of the lower limit defining the red sequence in Figure 1. Inspection of the distribution of blue cloud galaxies confirms that they have a very different phasespace distribution when compared to the red sequence galaxies, thus justifying our decision not to use them when estimating the virial mass. We note that our procedure rejects a number of galaxies which lie close to the boundaries defined by the maximum velocity profile but also appear to be well separated from the more clear-cut non-members. These consist primarily of blue cloud galaxies and, given their large peculiar velocities, 
are likely to be galaxies on radial orbits on their first passage onto the cluster. We prefer the more conservative cut here, since these galaxies appear to be distributed evenly in phase space and are thus unlikely to be part of the substructures we seek to detect, while their inclusion in the sample may affect the tests we plan to use for detecting substructure in the later sections.

The final cluster member catalogs contain 956 and 400 members for A2142 and RXJ1720, respectively. From the ensemble of member redshifts we use biweight estimators (Beers et al. 1990) to measure cluster redshifts of $z_{\text {clus }}=$ $0.09005 \pm 0.00012$ and $z_{\text {clus }}=0.16010 \pm 0.00018$ and peculiar velocity dispersions $\sigma_{\mathrm{vpec}}=995 \pm 21 \mathrm{~km} \mathrm{~s}^{-1}$ and $\sigma_{\text {vpec }}=882 \pm 29 \mathrm{~km} \mathrm{~s}^{-1}$ for A2142 and RXJ1720, respectively, where the uncertainties are $1 \sigma$ and are estimated using the jack-knife resampling approach (Beers et al. 1990).

\subsection{Substructure Detection}

Ultimately, our goal in this study is to test the hypothesis that cold fronts in clusters with otherwise regular $\mathrm{X}$-ray morphologies can be caused by the gravitational perturbation of an infalling substructure during a minor merger (Markevitch et al. 2001, Ascasibar \& Markevitch 2006, Markevitch \& Vikhlinin 2007, Owers et al. 2009b, Roediger et al. 2011). To that end, we use our samples of spectroscopically confirmed cluster members to search for merger-related substructures in the $1 \mathrm{D}$ velocity, $2 \mathrm{D}$ spatial and 3D velocity-plus-spatial distributions.

\subsubsection{Testing the shape of the peculiar velocity distribution}

A first order test of the dynamical regularity of a cluster comes from the shape of the peculiar velocity distribution which, in a dynamically relaxed cluster, is expected to take an approximately Gaussian shape. Gross departures from a Gaussian shape, particularly in the form of a skewness or bimodality, often indicate the existence of merger activity (e.g., Zabludoff et al. 1993, Owers et al. 2011). We choose to use the Gauss-Hermite reconstruction method (Zabludoff et al. 1993; see also Owers et al. 2009c) to test for departures from a Gaussian shape. Briefly, the velocity distribution is described by a series of Gauss-Hermite functions with the Gauss-Hermite moments $h_{0} \simeq 1$ multiplying the zeroth order term, which is the best fitting Gaussian with mean $V$ and standard deviation $S$, while the $h_{3}$ and $h_{4}$ terms approximate asymmetric and symmetric deviations from a Gaussian. Radial changes in the velocity distribution profile, e.g., the decrease in the velocity dispersion with radius observed in many clusters den Hartog \& Katgert 1996), can cause significant $h_{4}$ terms which are not related to merger activity. Our aim is to detect merger related substructure rather than non-Gaussian shapes caused by a changing velocity dispersion profile so, in addition to measuring the Gauss-Hermite distribution for the entire cluster member sample, we also repeat the GaussHermite reconstruction for members with $1500<R_{\text {proj }}<$ $3000,750<R_{\text {proj }}<1500$ and $R_{\text {proj }}<750 \mathrm{kpc}$. To determine the significance of the $h_{3,4}$ terms, we generate 10,000 Gaussian random distributions with the same number of data points, mean and standard deviation as the observed velocity distribution and measure the $h_{3,4}$ value for each random velocity distribution. We define
$P\left[h_{3,4}\right]=2 \min \left\{P\left[h_{3,4}(\mathrm{sim})<h_{3,4}(\mathrm{obs}), P\left[h_{3,4}(\mathrm{sim})>\right.\right.\right.$ $\left.\left.h_{3,4}(\mathrm{obs})\right]\right\}$ where $P\left[h_{3,4}(\mathrm{sim})<h_{3,4}(\mathrm{obs})\right]$ and $P\left[h_{3,4}(\mathrm{sim})>h_{3,4}(\mathrm{obs})\right]$ are determined from the number of times a $h_{3,4}$ value smaller and larger than the observed $h_{3,4}$ term is measured in the 10,000 random distributions. The results of this analysis are shown in Figure 5 for A2142 and Figure 6 for RXJ1720.

The A2142 velocity distributions for the radial ranges $R_{\text {proj }}<3000,1500<R_{\text {proj }}<3000$ kpc have significant positive $h_{3}$ terms. This indicates that the peak of the distribution is more negative and that the distribution has more power in the positive peculiar velocity tails than expected from the best-fitting Gaussian. The $1500<R_{\text {proj }} \leq 3000 \mathrm{kpc}$ velocity distribution also has a significant negative $h_{4}$ term, which indicates the velocity distribution has a broader, more boxy shape than the best fitting Gaussian. The velocity distribution in the range $750<R_{\text {proj }} \leq 1500 \mathrm{kpc}$ does not appear to have a shape which differs significantly from a Gaussian one, but we note that the measured velocity dispersion, $S=1184 \mathrm{~km} \mathrm{~s}^{-1}$ is larger than that measured at other radii. The peculiar velocity distribution in the central $750 \mathrm{kpc}$ returns marginally significant negative $h_{3}$ and positive $h_{4}$ values indicating that there are symmetric and asymmetric distortions present, although the marginal significances do not allow strong conclusions to be drawn.

The velocity distribution for the entire RXJ1720 member sample (top left panel in Figure 6) is well approximated by a Gaussian - small deviations are present at the $5-6 \%$ level in both the $h_{3}$ and $h_{4}$ terms but they are only significant at the $10 \%$ level. The velocity distribution for the cluster members with $1500<R_{\text {proj }}<3000 \mathrm{kpc}$, (top right panel in Figure 6) has a negative $h_{3}$ term which is significant at the $2 \%$ confidence level. This indicates that the velocity distribution in this radial bin has a peak which is shifted towards positive peculiar velocities and a tail with slightly more power at negative peculiar velocities. The remaining two velocity distributions (lower panels in Figure 6) do not harbor any significant deviations from a Gaussian shape.

\subsubsection{Substructure in the spatial distribution of galaxies}

Previous studies have shown that clusters in the process of merging may contain substructures which have little to no significant detectable impact on the shape of the peculiar velocity distributions, particularly in cases where the merger plane is perpendicular to our line of sight (Pinkney et al. 1996, Owers et al. 2009c a ). In these cases, the substructure may be conspicuous as local peaks in the projected surface density of the galaxies which indicate the existence of compact, bound subclusters. Here, we utilize our comprehensive spectroscopically confirmed cluster member samples to search for spatially compact substructures.

Clusters exhibit a large dynamic range in their projected galaxy surface density distributions. Therefore, in order to both resolve spatially close substructures and minimize the effects of noise in low density regions, an adaptive smoothing kernel is required. We use a Gaussian kernel with FWHM equal to the radius of the $N$ th nearest neighbor in projection, where $N=\sqrt{\left(n_{\text {mem }}\right)}$ and $n_{\mathrm{mem}}$ is the number of members in the sample, to smooth the projected galaxy distribution. The results 

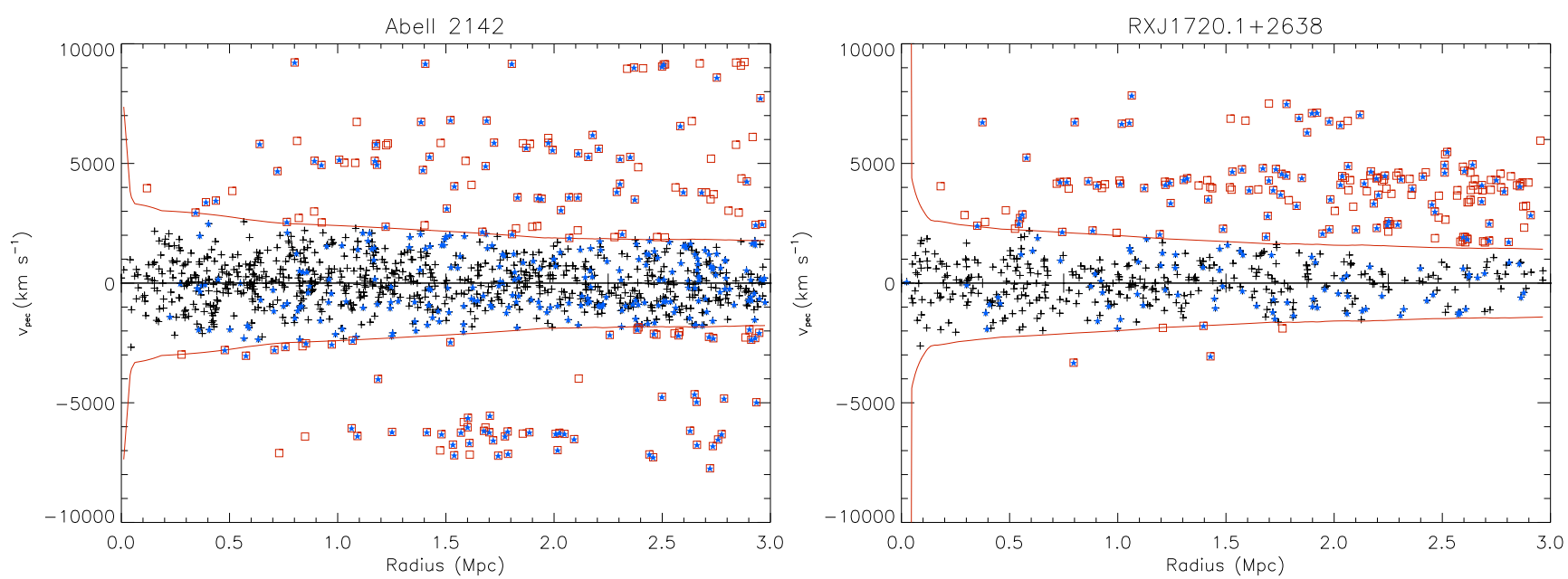

Figure 4. Phase space diagrams used for defining cluster membership for A2142 (left panel) and RXJ1720 (right panel). The red curves show the cluster limits in phase-space and are determined from the cluster mass profile (see the text for a description). Black crosses show cluster members which lie on the red sequence, while blue stars within the phase space limits show those cluster members which lie blueward of the red sequence. Red boxes show fore- and background galaxies. Red boxes filled with blue stars show non-members which are bluer than the cluster red sequence.
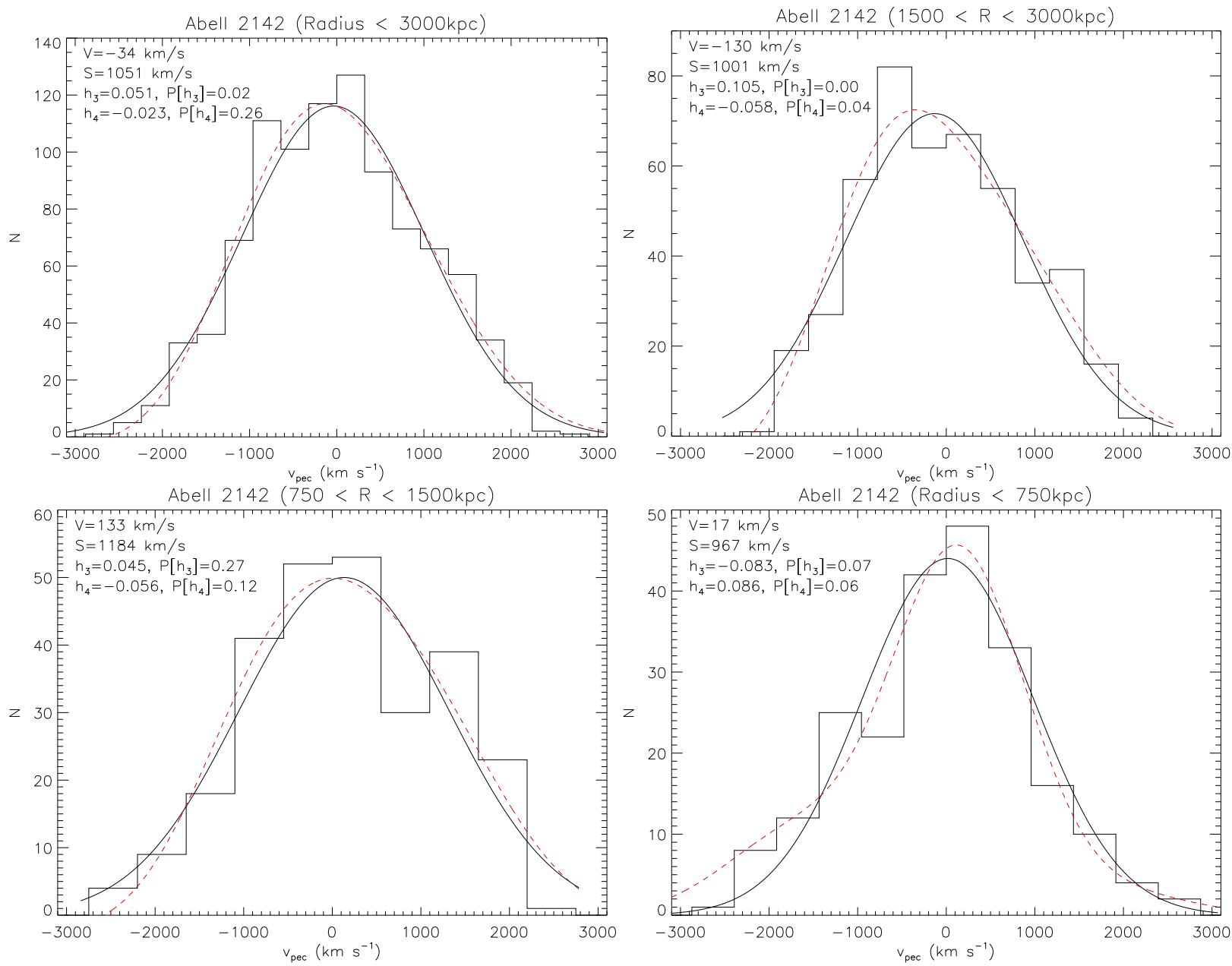

Figure 5. The velocity distribution for all 956 cluster members (top left panel), 463 members with $1500<R_{\text {proj }}<3000 \mathrm{kpc}$ (top right), 270 members with $750<R_{\text {proj }}<1500 \mathrm{kpc}$ (bottom left) and 223 members with $R_{\text {proj }}<750 \mathrm{kpc}$ (bottom right). In each panel, the best fitting Gaussian parameters, the Gauss-Hermite terms $h_{3}$ and $h_{4}$, representing the asymmetric and symmetric deviations from a Gaussian shape, and their associated level of significance (see text) are given in the upper right corner. The histograms show the observed velocity distribution and the bin size is set to $3 S /\left(N_{\text {gal }}\right)^{(1 . / 3 .)}$, where $N_{\text {gal }}$ is the number of galaxies listed above. The solid black line shows the best fitting Gaussian, while the dashed red line shows the Gauss-Hermite reconstriction of the velocity distribution. 

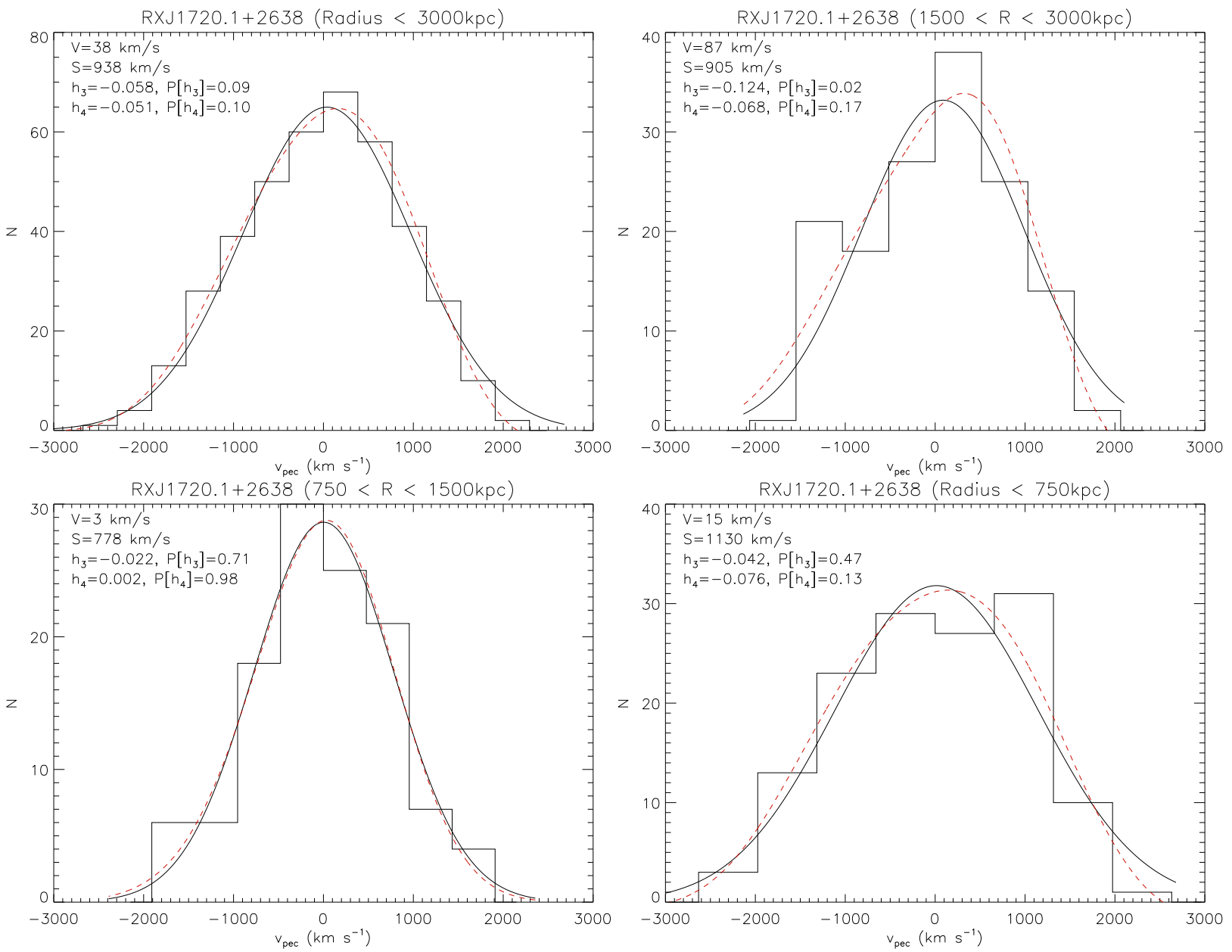

Figure 6. The same as Figure 5 but for RXJ1720. The number of galaxies in each subsample shown in the top left, top right, bottom left and bottom right are 400, 146, 117 and 137 galaxies, respectively. 
of the smoothing are shown in the top left panels of Figures 7 and 8 for A2142 and RXJ1720, respectively. Both clusters exhibit a dense core of galaxies surrounding the central BCG, elongated morphologies on larger scales and a number of local overdensities. The central regions of A2142 appear to be irregular, with a number of local overdensities within $1 \mathrm{Mpc}$ of the cluster center, the most significant of which occurs $\sim 180 \mathrm{kpc}$ to the NW, roughly coincident with the second BCG. There are also four large conglomerations of galaxies in the outskirts of A2142; one $\sim 2.4 \mathrm{Mpc}$ to the SE, one $\sim 1.7 \mathrm{Mpc}$ due south, one $\sim 2.5 \mathrm{Mpc}$ to the SW and one $2.2 \mathrm{Mpc}$ to the NW. Most notably for RXJ1720, there is a second peak in the projected galaxy density slightly east of due north and $\sim 550 \mathrm{kpc}$ from the cluster center, while there is a less significant overdensity $\sim 380 \mathrm{kpc}$ to the SW. There is also an overdensity $\sim 1.7 \mathrm{Mpc}$ just west of south in the outskirts of RXJ1720.

\subsubsection{Substructure in the local kinematics}

Having identified evidence for substructure in both the velocity and spatial distributions, we now search for correlations between the local kinematics and the projected local overdensities in the galaxy distribution. As a first attempt at this, we utilize the $\kappa$-test which identifies kinematic substructures by comparing the local velocity distribution of the $N=\sqrt{n_{\mathrm{mem}}}$ nearest neighbors around each galaxy to the global velocity distribution (Colless \& Dunn 1996). The comparison is quantified using the Kolmogorov-Smirnov (KS) test, which returns the likelihood, $P_{\mathrm{KS}}$, that the local and global velocity distributions are drawn from the same parent distribution. The overall level of substructure within the cluster can be quantified by summing the individual $-\log \left[P_{\mathrm{KS}}\right]$ to obtain the $\kappa$-statistic. The significance of the $\kappa$-statistic is obtained by comparing the observed value to the distribution of 10,000 remeasurements of the $\kappa$-statistic which have been obtained from samples where the member velocities are randomly redistributed amongst the positions, which are held fixed, thus erasing coherent dynamical substructure. For A2142 and RXJ1720 we measure $\kappa$-statistics of 836 and 256 at a significance of $5.6 \sigma$ and $2.5 \sigma$, respectively, indicating that A2142 harbors significant dynamical substructure, while RXJ1720 shows evidence for dynamical substructure, although at a lower significance level.

The results of the $\kappa$-test for the full field of view for A2142 and RXJ1720 are shown in the top right panels of Figures 7 and 8 respectitvely. At the position of each member galaxy, we plot a circle with radius $R \propto-\log \left[P_{\mathrm{KS}}\right]$. Each circle is color coded so that members with positive $v_{\text {pec }}$ are red while members with negative $v_{\text {pec }}$ are blue. Furthermore, those members having $-\log \left[P_{\mathrm{KS}}\right]$ values occurring in only $5 \%$ of the random realizations are plotted with thick lines. Clusterings of these large, emboldened circles indicate that there exists localized velocity substructure. For comparison to the substructures revealed in Section 3.2.2 we also overplot the corresponding surface density contours.

Within a $1 \mathrm{Mpc}$ radius of the center of A2142 (left panel of Figure 9), there is one region of large clustered circles $\sim 1 \mathrm{Mpc}$ to the NW which is coincident with an extension in the galaxy surface density contours. There is no significant clustering of large circles coincident with the local galaxy overdensity associated with the second $\mathrm{BCG} \sim 180 \mathrm{kpc}$ to the northwest. We note, however, that this does not rule out the existence of a compact dynamical substructure here, particularly given the enhanced projection effects due to the close proximity to the main cluster core which serve to dilute differences in the velocity distribution of a real substructure and the main cluster. In the cluster outskirts (top right panel of Figure 7), there are three concentrations of large circles, two of which coincide approximately with local overdensities in the spatial distribution of galaxies. The most significant of these is located $\sim 2.6 \mathrm{Mpc}$ to the northwest and contains a large number of members with negative $v_{\text {pec }}$. The second lies $\sim 2.4 \mathrm{Mpc}$ to the $\mathrm{SE}$, while the third, which is not associated with a local overdensity in the galaxy distribution, lies $\sim 2.3 \mathrm{Mpc}$ due west. There is a fourth region $\sim 2.4 \mathrm{Mpc}$ to the $\mathrm{SW}$ and coinciding with a local overdensity in the galaxy distribution which contains a more mild concentration of large circles. Further investigation of this region is required to confirm the existence of dynamical substructure here.

Considering the central $1 \mathrm{Mpc}$ region of RXJ1720 (right panel in Figure 9) it can be seen that there is a clustering of large circles associated with the prominent secondary galaxy surface density substructure located $\sim 550 \mathrm{kpc}$ to the north of the cluster center. There is also a smaller clustering of large circles $\sim 380 \mathrm{kpc}$ to the SW which is coincident with one of the more minor substructures in the galaxy surface density distribution, indicating there is dynamical substructure there. At larger radii (top right panel of Figure 8), we note that there are a number of larger circles associated with the galaxy overdensity $\sim 1.7 \mathrm{Mpc}$ just west of south, although only a fraction of those are deemed to be significantly larger than expected, indicating that further investigation is required before concluding that there is local dynamical substructure here.

The $\kappa$-test is an ideal tool for detection of substructure but provides little information on kinematic properties which are key to understanding and characterizing substructure. For the purpose of characterizing these substructures, we produce maps tracing the mean velocity and velocity dispersion fields which are presented in the lower panels of Figures 7 and 8 for A2142 and RXJ1720, respectively. Briefly, these maps were produced by generating a grid of $50 \times 50 \mathrm{kpc}$ pixels and determining at each pixel the weighted mean, $\overline{v_{w}}$,

$$
\overline{v_{w}}=\frac{\sum_{i=1}^{N} w_{i} v_{\mathrm{pec}, i}}{\sum_{i=1}^{N} w_{i}}
$$

and dispersion, $\sigma\left(v_{w}\right)$,

$$
\sigma\left(v_{w}\right)^{2}=\frac{\sum_{i=1}^{N} w_{i}\left(v_{\mathrm{pec}, i}-\overline{v_{w}}\right)^{2}}{\sum_{i=1}^{N} w_{i}}
$$

of the $N=\sqrt{n_{\text {mem }}}$ nearest neighbors, where $v_{\text {pec }, i}$ is the peculiar velocity of the $i$ th near neighbor, $w_{i}=1-$ $\left(r_{i} / R\right), r_{i}$ is the radial distance of the $i$ th nearest member 

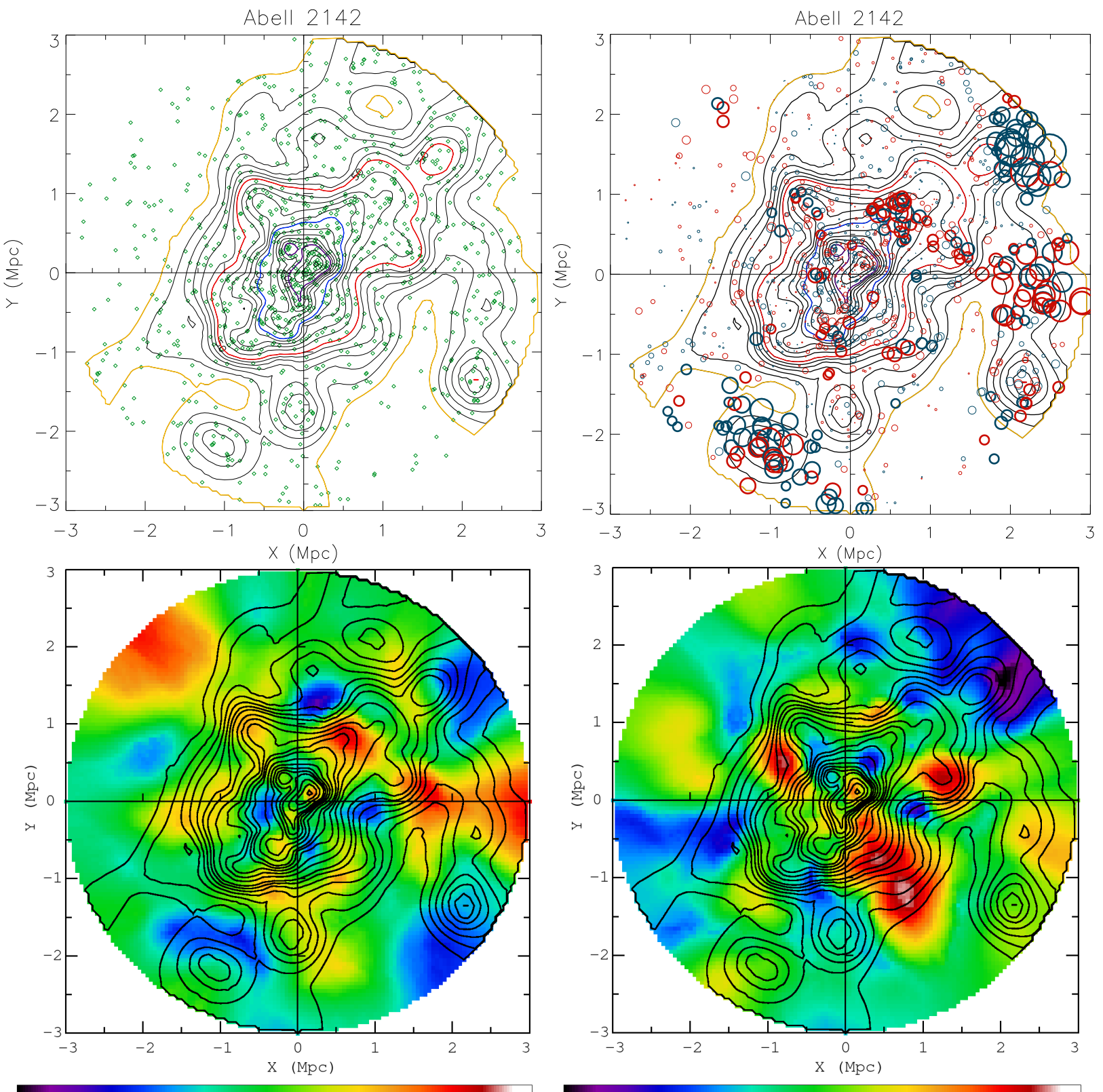

$1000-800-600-400-200 \quad 0 \quad 200 \quad 400 \quad 600 \quad 800 \quad 100400 \quad 500 \quad 600 \quad 700 \quad 800 \quad 900 \quad 10001100120013001400150$

Figure 7. Top left panel: Isopleths showing the galaxy surface density distribution. The levels are not spaced equally, but are spaced at intervals of 5, 10, 20 and 50 galaxies/ $\mathrm{Mpc}^{2}$ between the levels 20-50, 50-100, 100-200 and above 200 galaxies/Mpc ${ }^{2}$, respectively. The 20, 50, 100 and 200 galaxies $/ \mathrm{Mpc}^{2}$ levels are colored yellow, red, light blue and dark blue, respectively. Green diamonds show the member positions. Top right panel: Results of the $\kappa$-test where the circle size gives an indication of the difference of local velocity distribution compared to the global cluster velocity distribution. Clusterings of large emboldened circles indicate significant departures. Blue and red circles are centered on galaxies which have negative and positive peculiar velocities, respectively. Bottom panels: Maps of the mean velocity (left) and velocity dispersion (right) fields generated using Equations 1 and 2 The units of the values on the colorbars are $\mathrm{km} \mathrm{s}^{-1}$. Contours in the top right and bottom panels are the same as those shown in the top right panel.

from the pixel center and $R=r_{i=N}$.

Examining the results for A2142 in the bottom two panels of Figure 7 we note that there is a general coincidence of regions which contain clustered large circles seen in the $\kappa$-test bubble plot and regions where either one or both of the $\overline{v_{w}}$ and $\sigma\left(v_{w}\right)$ fluctuate away from the overall cluster values. The region of clustered large circles $\sim 1 \mathrm{Mpc}$ to the NW coincides with a region where $\overline{v_{w}} \sim 750 \mathrm{~km} \mathrm{~s}^{-1}$ while the most significant clustering of large bubbles located $\sim 2.6 \mathrm{Mpc}$ to the NW coincides with a region where $\overline{v_{w}} \sim-600 \mathrm{~km} \mathrm{~s}^{-1}$ and $\sigma\left(v_{w}\right) \sim 400 \mathrm{~km} \mathrm{~s}^{-1}$. The substructure $\sim 2.4 \mathrm{Mpc}$ to the SE has mainly negative $\overline{v_{w}}$ values which peak at $\overline{v_{w}} \sim-550 \mathrm{~km} \mathrm{~s}^{-1}$ just to the north of the local peak in the galaxy density. Similarly, the velocity field surrounding the local galaxy overdensity $\sim 2.5 \mathrm{Mpc}$ to the southwest has values of $\overline{v_{w}} \sim-450 \mathrm{~km} \mathrm{~s}^{-1}$, while the substructure $\sim 2.5 \mathrm{Mpc}$ due west has $\overline{v_{w}} \sim 500 \mathrm{~km} \mathrm{~s}^{-1}$. These maps also reveal that the kinematics immediately surrounding the second BCG $\sim 180 \mathrm{kpc}$ NW of the cluster center are different, with $\overline{v_{w}} \sim 500 \mathrm{~km} \mathrm{~s}^{-1}$ and $\sigma\left(v_{w}\right) \sim 1200 \mathrm{~km} \mathrm{~s}^{-1}$. 

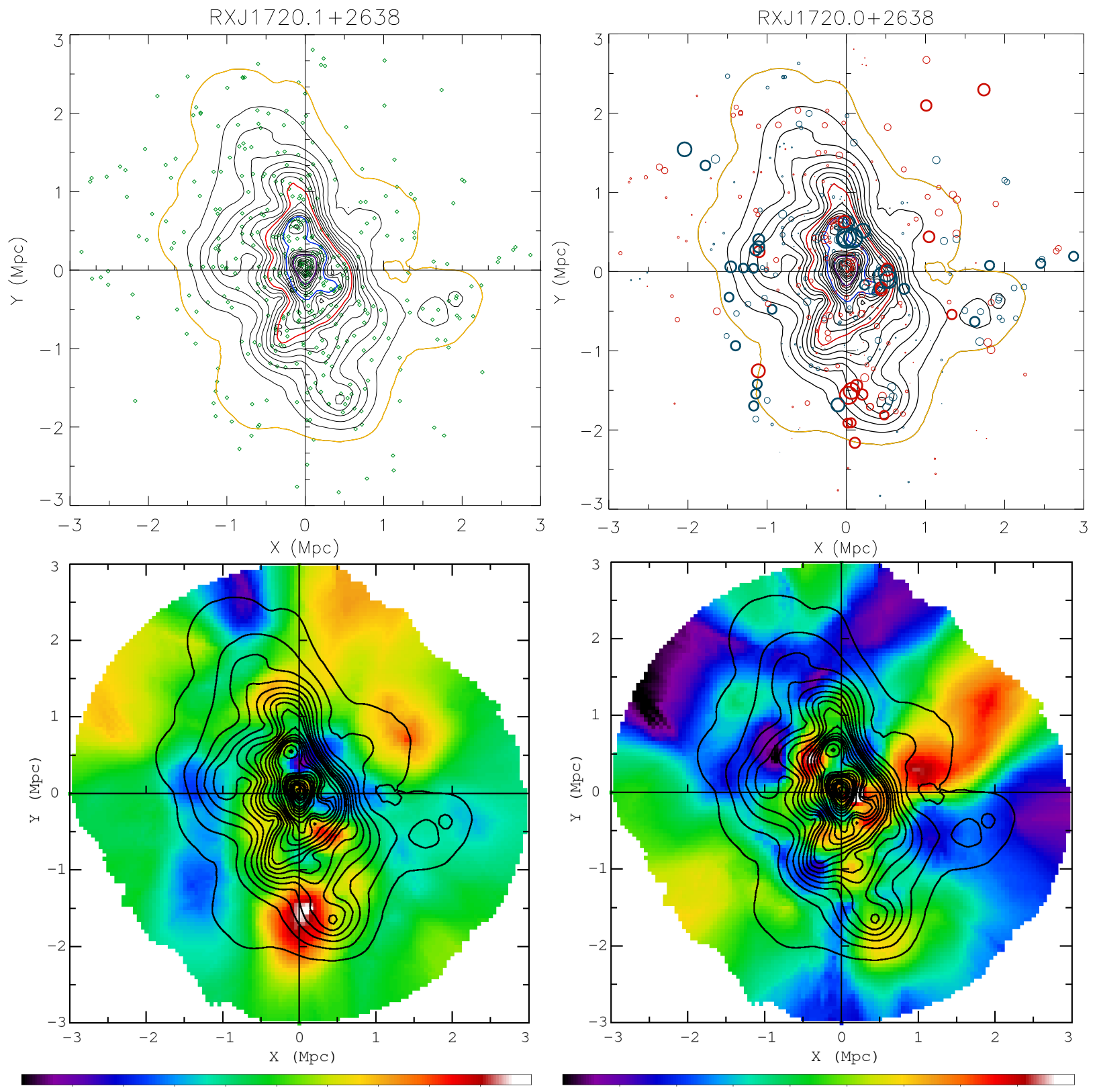

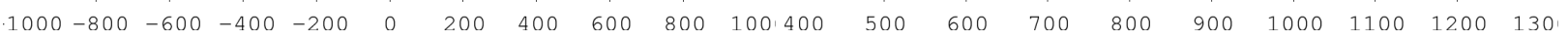

Figure 8. The same as Figure 7 but for RXJ1720. Here, the lowest isopleth is 10 galaxies $/ \mathrm{Mpc}^{2}$.

For RXJ1720 (Figure 8) we find a general agreement between the regions which have fluctuations in either one or both of $\overline{v_{w}}$ and $\sigma\left(v_{w}\right)$ and regions containing the most significant local departures from the global velocity distribution according to the $\kappa$-test. The region surrounding the local projected galaxy overdensity located $\sim 550 \mathrm{kpc}$ north of the cluster center shows very complex velocity structure. The regions just SW and NE of this substructure have $\overline{v_{w}} \simeq-800 \mathrm{~km} \mathrm{~s}^{-1}$ and $\simeq 250 \mathrm{~km} \mathrm{~s}^{-1}$, respectively, while the the region immediately to the $\mathrm{SE}$ of this substructure has $\sigma\left(v_{w}\right) \simeq 1200 \mathrm{~km} \mathrm{~s}^{-1}$. The velocity field coincident with the substructure $\sim 400 \mathrm{kpc}$ to the SW has $\overline{v_{w}} \simeq 450 \mathrm{~km} \mathrm{~s}^{-1}$, while the velocity field surrounding the local galaxy overdensity $\sim 1.7 \mathrm{Mpc}$ to the south has $\overline{v_{w}} \sim 400 \mathrm{~km} \mathrm{~s}^{-1}$, and shows a peak of $v_{w} \sim 900 \mathrm{~km} \mathrm{~s}^{-1}$ just to the east of the local peak in the galaxy density.
The $\kappa$-test and the velocity field maps described above are ideal for identifying local kinematic substructure and go some way towards classifying the dynamical properties of the substructure. However, the data at hand allow us to go one step further in the process of visualizing and characterizing substructure by producing "tomograms" of the galaxy density in peculiar velocity slices. These tomograms are shown in Figures 10 and 11 for A2142 and RXJ1720, respectively. We produce maps at nine central velocities where the $x-y-v_{\text {pec }}$ distribution has been smoothed with a 3D Gaussian Kernel with an adaptively varying $\sigma_{x y}$ (the same kernel described in Section 3.2.2) and $\sigma_{v}=300 \mathrm{~km} \mathrm{~s}^{-1}$. The central velocities used in the smoothing are listed in the top left corners of Figures 10 and 11 which show contours of the 3D galaxy density in units of galaxies per $\mathrm{Mpc}^{2}$ 

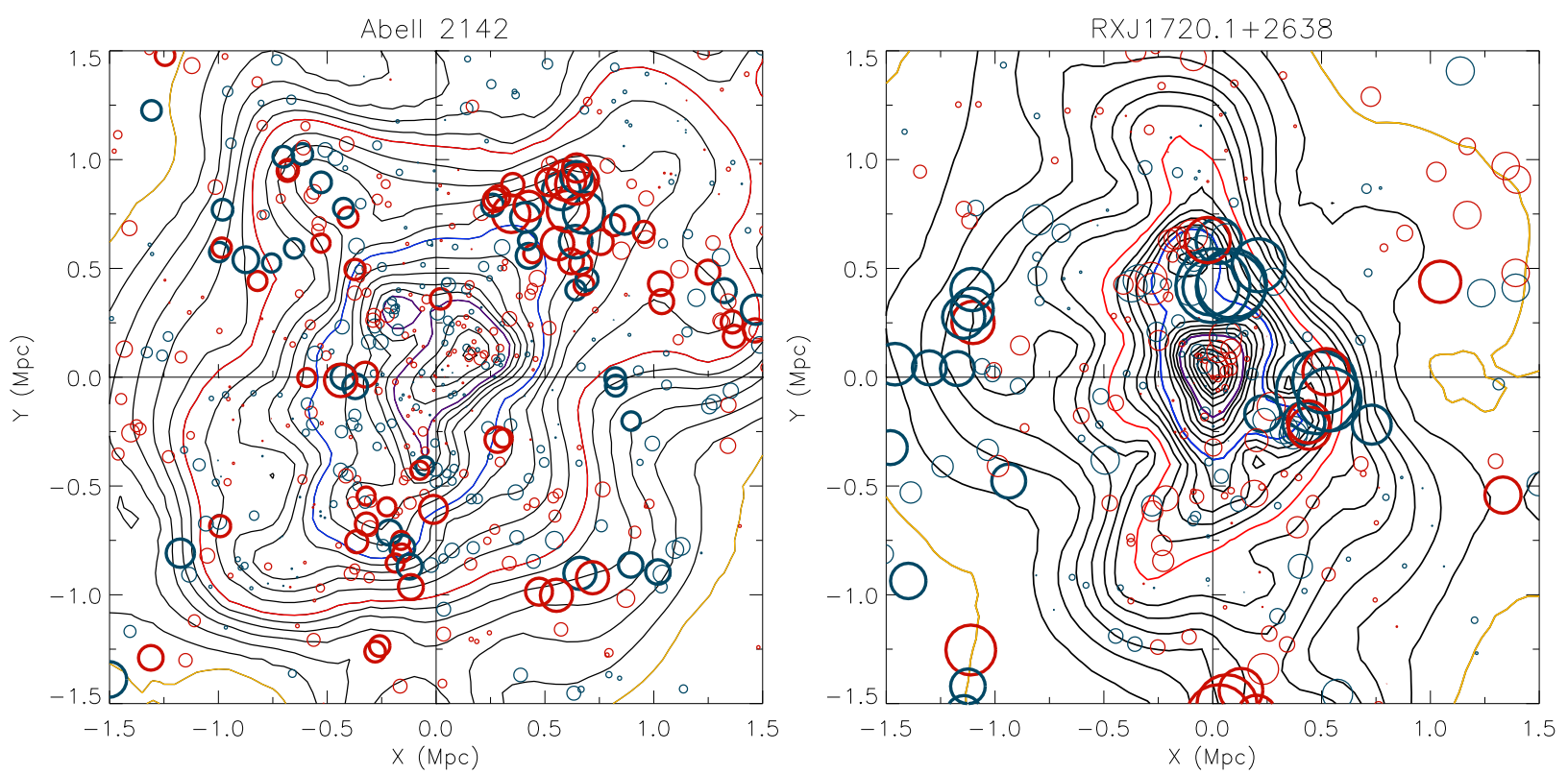

Figure 9. Zoomed version of the top right panels in Figures 7 and 8 showing the central $1.5 \mathrm{Mpc}$ regions.

per $1000 \mathrm{~km} \mathrm{~s}^{-1}$. The central core is the dominant feature in all of the tomograms for RXJ1720 (Figure 11) which confirm the complex nature of the dynamics surrounding the local galaxy overdensity located $\sim 550 \mathrm{kpc}$ to the $\mathrm{N}$ of the core - it appears most significant in the $\bar{v}_{\text {pec }}=-1000,-500,500$ and $1000 \mathrm{~km} \mathrm{~s}^{-1}$ maps, but is relatively insignificant in the $\bar{v}_{\text {pec }}=0 \mathrm{~km} \mathrm{~s}^{-1}$ map. Unlike in RXJ1720, the core does not dominate in all of the tomograms for A2142 (Figure 10) which are also more complicated than their counterparts in RXJ1720. Comparison of the tomograms with those maps presented in Figure 7 reveals that those substructures seen in the cluster outskirts generally appear to be fairly well isolated structures in velocity space, too. The tomograms show that the conglomeration of significant circles $\sim 1 \mathrm{Mpc}$ to the NW of the core appears as a feature in the $\bar{v}_{\text {pec }}>500 \mathrm{~km} \mathrm{~s}^{-1}$ maps, while the local overdensity associated with the second BCG appears most prominently at $\bar{v}_{\text {pec }}=1500$ and $2000 \mathrm{~km} \mathrm{~s}^{-1}$.

\subsubsection{Characterizing the substructures with KMM}

Having identified substructure with a number of techniques in Sections 3.2 .2 and 3.2.3, we are now in a position to characterize the dynamical properties of the substructures. To achieve this, we use the Kaye's Mixture Model algorithm of Ashman et al. (KMM; 1994) to investigate the dynamics within the regions shown in Figures 12 and 13. The sizes of these regions were chosen to contain $\sim 1.5 \sqrt{N_{\mathrm{mem}}}=50$ and 30 nearest neighbors for A2142 and RXJ1720, respectively. For each of these regions, the KMM algorithm was used to fit a minimum of two Gaussians to the velocity distribution. The first Gaussian component models the main cluster velocity distribution while the remaining Gaussian components model the superimposed substructure velocity distribution. The KMM algorithm requires initial estimates of the mean $(\mu)$, variance $\left(\sigma^{2}\right)$ and the fraction of galaxies $(f)$ belonging to each Gaussian velocity component. The initial estimate for the main cluster component is set to $\left(\mu_{1, \text { in }}, \sigma_{1, \text { in }}, f_{1, \text { in }}\right)=\left(0 \mathrm{~km} \mathrm{~s}^{-1}, 1000 \mathrm{~km} \mathrm{~s}^{-1}, 0.8\right)$, while the initial estimates for the remaining Gaussian components are determined by inspection of the velocity distribution and are listed in Tables 5 and 6 . We determine the significance of each bimodal fit by employing a parametric Bootstrap method (see Owers et al. 2011). Briefly, we produce 1000 random resamplings of a Gaussian with 50 (for A2142) or 30 (for RXJ1720) data points and $\mu$ and $\sigma$ set to the values derived from the entire cluster sample. The KMM algorithm is then used to fit two Gaussians to the resampled distribution with the initial inputs set to the outputted KMM fits to the observed data. A measure of the improvement of the fit in going from a unimodal to bimodal Gaussian distribution is given by the Likelihood Ratio Test Statistic (LRTS; see Ashman et al. 1994). The distribution of LRTSs produced by the resampled distributions can be compared to the observed LRTS to determine if the results of the fit to the observed distribution can be caused by random fluctuations in the data and this is listed as a $P$-value in Table 5 and 6 In a number of cases (e.g., S5 and S6 for A2142 and S1 for RXJ1720) a three-mode partition was also fitted to the data. For these cases, the $P$-value is evaluated by comparison of the trimodal fit to the bimodal one.

\subsection{Summary of the substructure properties}

Having identified many significant substructures in both A2142 and RXJ1720, and characterized them dynamically using the KMM method, we now briefly discuss each of them and, in particular, their properties that are most pertinent to our study.

\subsubsection{A2142}

- S1: This substructure is coincident with the second BCG $\sim 180 \mathrm{kpc}$ to the northwest of the central BCG. There is a local overdensity in the projected galaxy distribution here, although the $\kappa$ test does not reveal strong evidence that the local velocity distribution differs significantly from the global one. The substructure is prominent as 


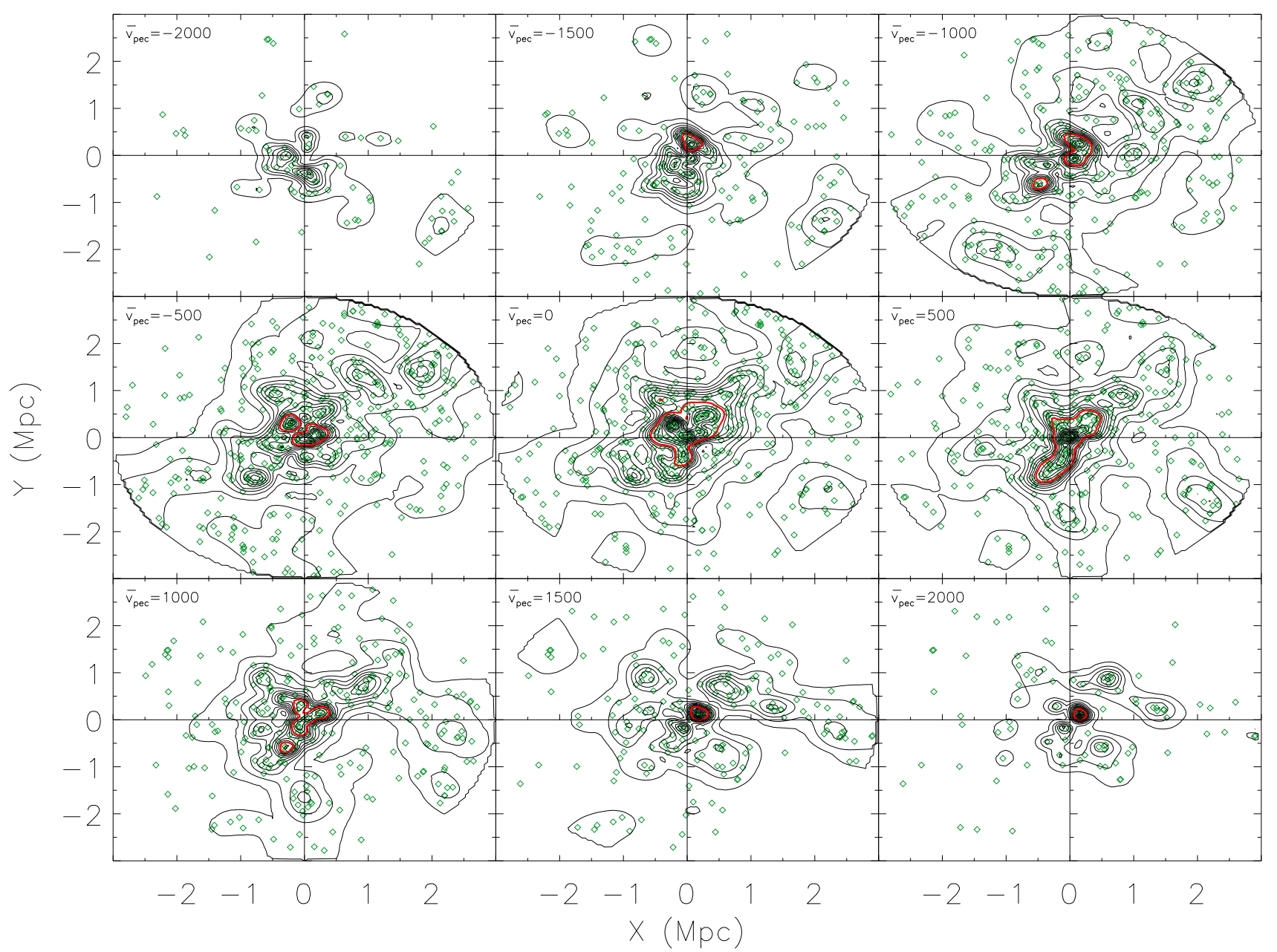

Figure 10. Tomograms for A2142 revealing overdensities in position-velocity space centered at the velocities listed in the upper left of each panel. The spatial smoothing uses the same adaptive kernel as that used in the top left panel of Figure 7 while the velocity smoothing uses a fixed sigma of $300 \mathrm{~km} \mathrm{~s}^{-1}$. The green diamonds show the positions of galaxies lying within $\pm 600 \mathrm{~km} \mathrm{~s}{ }^{-1}$ of the central velocity, $\bar{v}_{\text {pec }}$. The contours show the galaxy density and start at 5 galaxies $/ \mathrm{Mpc}^{2} /\left(1000 \mathrm{~km} \mathrm{~s}^{-1}\right)$ with an interval of 5 galaxies $/ \mathrm{Mpc}^{2} /\left(1000 \mathrm{~km} \mathrm{~s}{ }^{-1}\right)$ up to the 50 galaxies $/ \mathrm{Mpc}^{2} /\left(1000 \mathrm{~km} \mathrm{~s}^{-1}\right)$ level (red contour). Above 50 galaxies $/ \mathrm{Mpc}^{2} /\left(1000 \mathrm{kms}^{-1}\right)$, for clarity, the interval is 10 galaxies $/ \mathrm{Mpc}^{2} /\left(1000 \mathrm{~km} \mathrm{~s}^{-1}\right)$.

Table 5

KMM fits to the regions shown in Figure 12 for A2142.

\begin{tabular}{|c|c|c|c|c|c|}
\hline Region & $\begin{array}{c}(X, Y) \\
(\mathrm{kpc}, \mathrm{kpc})\end{array}$ & $\begin{array}{l}\text { Substructure Input } \\
\qquad(\mu, \sigma, f)\end{array}$ & $\begin{array}{l}\text { Main output } \\
\quad(\mu, \sigma, f)\end{array}$ & $\begin{array}{l}\text { Substructure output } \\
(\mu, \sigma, f)\end{array}$ & $\mathrm{P}$-value \\
\hline $\begin{array}{l}\text { S1 } \\
\text { S2 } \\
\text { S3 } \\
\text { S4 } \\
\text { S5 } \\
\text { S5 } \\
\text { S6 } \\
\text { S6 } \\
\text { S7 }\end{array}$ & $\begin{array}{c}(150,90) \\
(600,763) \\
(2007,1567) \\
(-1255,-2038) \\
(2072,-1494) \\
(2072,-1494) \\
(2327,-180) \\
(2327,-180) \\
(-1725,-100)\end{array}$ & $\begin{array}{c}(1800,300,0.2) \\
(1500,300,0.2) \\
(-600,400,0.2) \\
(-900,300,0.2) \\
(-1500,300,0.2) \\
(-1500,300,0.2),(600,200,0.1) \\
(1200,300,0.2) \\
(1200,300,0.2),(-400,200,0.1) \\
(900,300,0.2)\end{array}$ & $\begin{array}{c}(-232.8,938.7,0.86) \\
(186.3,638.3,0.82) \\
(500.1,688.7,0.18) \\
(114.7,1047.5,0.54) \\
(272.0,649.9,0.72) \\
(24.2,765.6,0.44) \\
(-701.1,522.5,0.40) \\
(-1212.4,423.8,0.14) \\
(-185.0,815.2,0.78)\end{array}$ & $\begin{array}{c}(1732.5,224.2,0.14) \\
(1681.0,368.8,0.18) \\
(-450.8,442.5,0.82) \\
(-913.4,178.5,0.46) \\
(-1608.3,153.1,0.28) \\
(-1620.4,144.1,0.26),(658.8,68.9,0.2) \\
(1156.5,357.8,0.60) \\
(1147.8,362.8,0.60),(-433.7,220.7,0.26) \\
(902.6,172.2,0.22)\end{array}$ & $\begin{aligned} & 0.434 \\
& 0.007 \\
< & 0.001 \\
< & 0.001 \\
< & 0.001 \\
& 0.007 \\
< & 0.001 \\
& 0.32 \\
& 0.11\end{aligned}$ \\
\hline
\end{tabular}




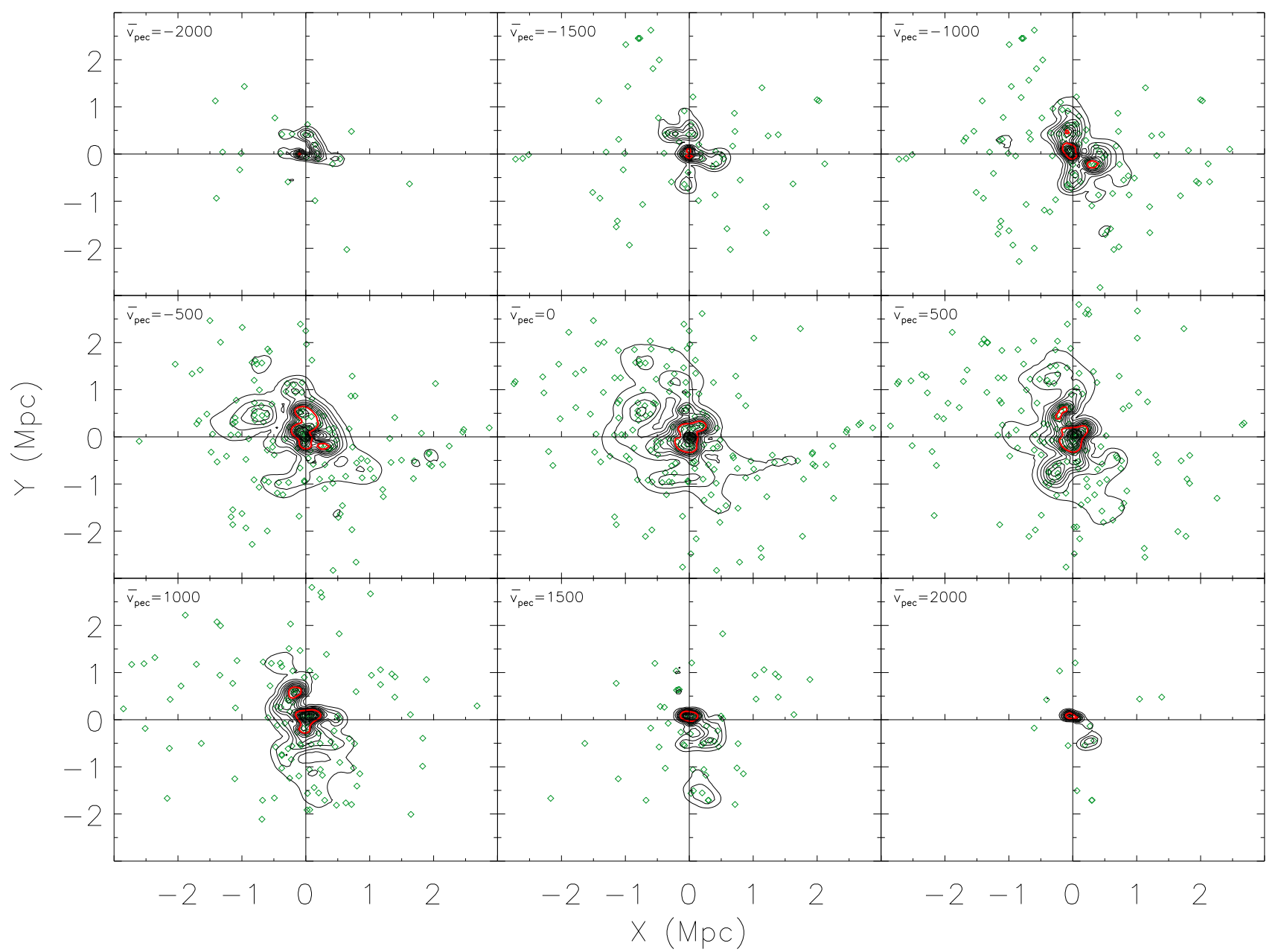

Figure 11. As for Figure10 but for RXJ1720. The spatial smoothing uses the same adaptive kernel as that used in the top left panel of Figure 8

Table 6

KMM fits to the regions shown in Figure 13 for RXJ1720.

\begin{tabular}{cccccc}
\hline \hline Region & $\begin{array}{c}(X, Y) \\
(\mathrm{kpc}, \mathrm{kpc})\end{array}$ & $\begin{array}{c}\text { Substructure Input } \\
(\mu, \sigma, f)\end{array}$ & $\begin{array}{c}\text { Main output } \\
(\mu, \sigma, f)\end{array}$ & $\begin{array}{c}\text { Substructure output } \\
(\mu, \sigma, f)\end{array}$ \\
\hline S1 & $(-50,576)$ & $(700,300,0.2)$ & $(-849.7,594.4,0.67)$ & $(736.0,184.2,0.33)$ \\
S1 & $(-50,576)$ & $(700,300,0.2),(-700,300,0.2)$ & $(-906.0,659.9,0.50)$ & $(733.8,185.6,0.33),(-658.2,127.9,0.17)$ & 0.72 \\
S2 & $(443,-144)$ & $(-900,300,0.2)$ & $(-42.4,1145.8,0.77)$ & $(-851.0,99.2,0.23)$ & 0.082 \\
S3 & $(302,-1706)$ & $(800,300,0.2)$ & $(-819.8,404.8,0.23)$ & $(818.4,512.5,0.76)$ & 0.009 \\
S4 & $(-1225,181)$ & $(-400,300,0.2)$ & $(-,-,-)$ & $(-291.1,577.3,1.00)$ & - \\
\hline
\end{tabular}



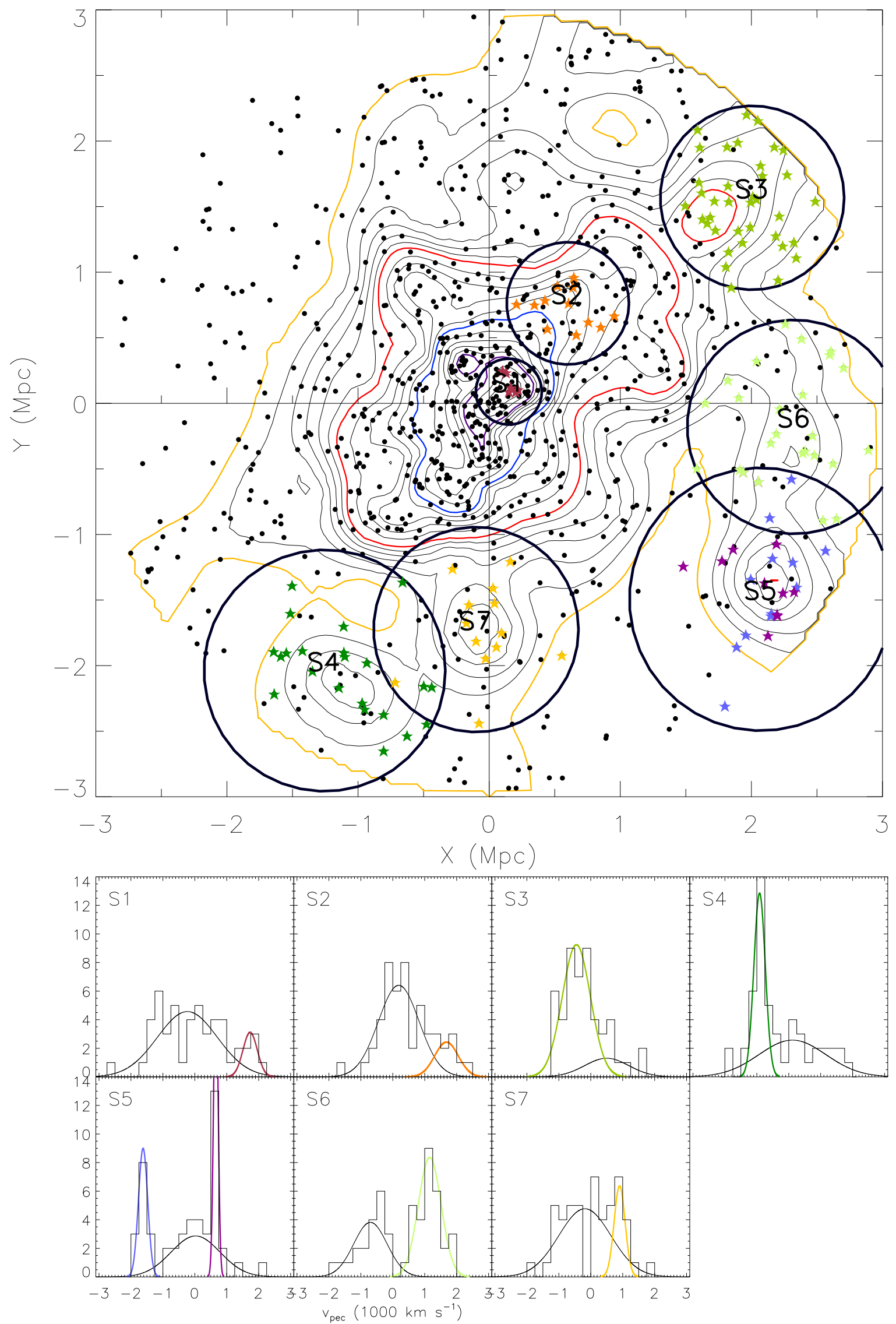

Figure 12. Top panel: The black circles show regions selected for the KMM analyses based on substructure revealed in Figure 7 and 10 Black contours show galaxy surface density as in Figure 7 The stars are color coded to match the corresponding velocity components shown in the lower panels. Black points reveal the positions of the remaining cluster members. 

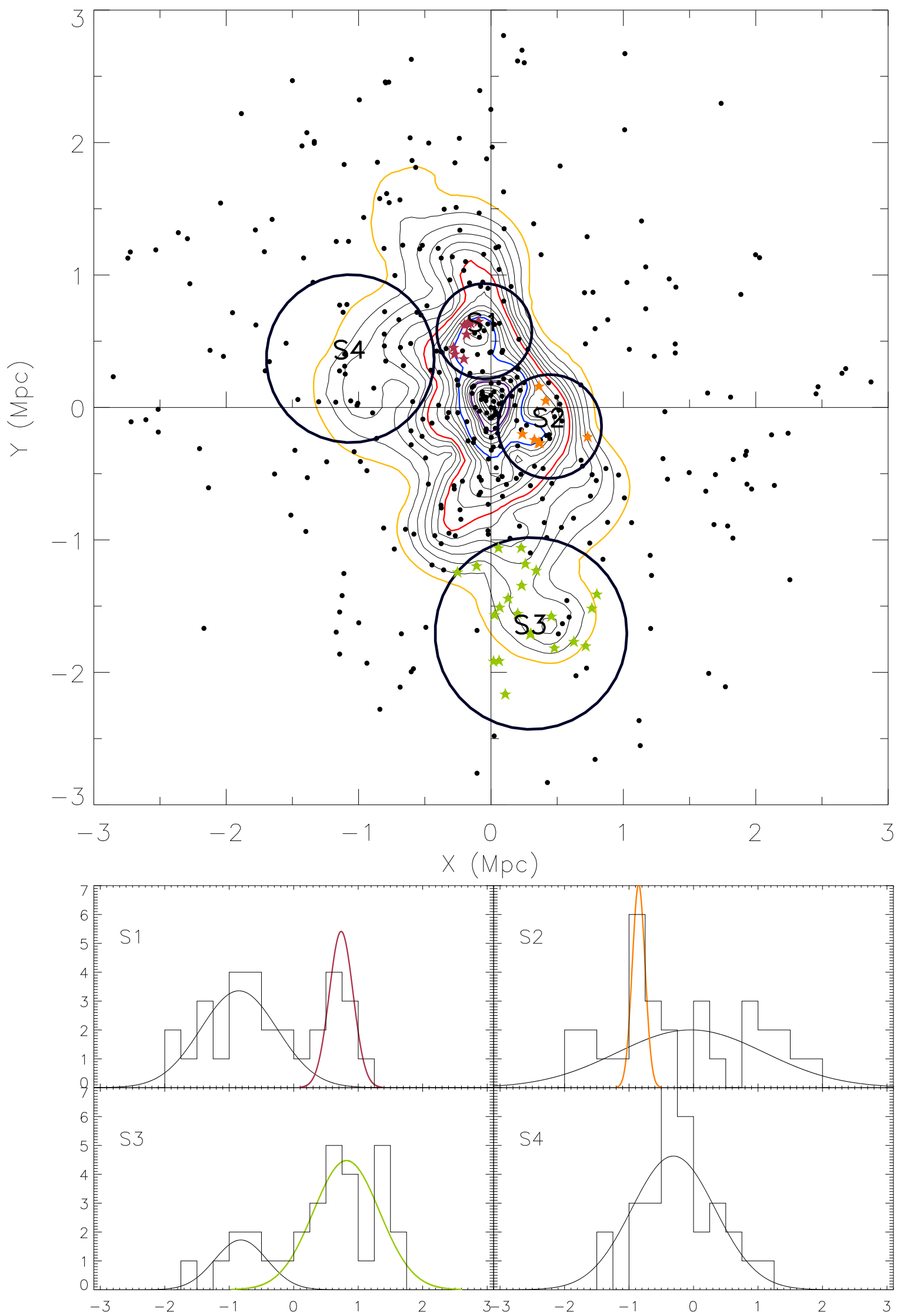

Figure 13. As for Figure 12 but for RXJ1720. 
an overdensity in the $\bar{v}_{\text {pec }}=1500$ and $2000 \mathrm{~km} \mathrm{~s}^{-1}$ tomograms, consistent with a grouping of galaxies around the velocity of the second BCG $\left(v_{\mathrm{pec}}=\right.$ $1760 \mathrm{~km} \mathrm{~s}^{-1}$ ). The KMM analysis returns a mean $v_{\text {pec }}=1732.5 \mathrm{~km} \mathrm{~s}^{-1}$ and dispersion $\sigma_{\text {vpec }}=$ $224.2 \mathrm{~km} \mathrm{~s}^{-1}$ for a group of $\sim 7-10$ galaxies surrounding this substructure, although a bimodal fit is not favored. The lack of evidence for dynamical substructure in the $\kappa$ - and KMM tests is probably due to S1's proximity to the cluster core where projection effects caused by the main cluster population are large. This is likely the remnant core of a more massive system which has had its less well bound members stripped during its infall.

- S2: This is a more loose agglomeration of spirals. There doesn't appear to be a dominant early type member within this substructure and there is only a mild excess in the galaxy surface density here. The velocity field is significantly positive in this region and the tomograms reveal local overdensities here which are most prominent at $\bar{v}_{\text {pec }}=1000$ and $1500 \mathrm{~km} \mathrm{~s}^{-1}$. The KMM fit reveals a significant bimodality where the substructure has mean velocity $v_{\text {pec }}=1681.0 \mathrm{~km} \mathrm{~s}^{-1}$ and dispersion of $\sigma_{\text {vpec }}=368.8 \mathrm{~km} \mathrm{~s}^{-1}$. Okabe \& Umetsu (2008) find a mildly significant peak in their projected mass maps at approximately the same position.

- S3: This substructure is clearly distinguished by the $\kappa$-test. It lies $\sim 2.5 \mathrm{Mpc}$ from the cluster center and it is conspicuous as a local overdensity in the projected galaxy distribution, as features in the velocity field maps and as an overdensity in the $\bar{v}_{\text {pec }}=-500 \mathrm{~km} \mathrm{~s}^{-1}$ tomogram. The KMM analysis returns significant bimodality and the substructure has mean velocity $v_{\text {pec }}=-450.8 \mathrm{~km} \mathrm{~s}^{-1}$ and dispersion $\sigma_{\mathrm{vpec}}=442.5 \mathrm{~km} \mathrm{~s}^{-1}$, consistent with a large group or small cluster.

- S4: Another substructure in the outskirts located $\sim 2.4 \mathrm{Mpc}$ from the center. This substructure manifests itself as a local overdensity in the projected galaxy distribution and in the $\bar{v}_{\text {pec }}=-1000 \mathrm{~km} \mathrm{~s}^{-1}$ tomogram. The KMM test reveals significant bimodality and the substructure has mean velocity $v_{\text {pec }}=-913.4 \mathrm{~km} \mathrm{~s}^{-1}$ with dispersion $\sigma=$ $178.5 \mathrm{~km} \mathrm{~s}^{-1}$, consistent with a group-sized substructure.

- S5: This substructure is a significant local overdensity in the projected galaxy distribution although the $\kappa$-test does not show conclusive results for local velocity substructure. The substructure is seen as an overdensity in the $\bar{v}_{\text {pec }}=-1500$ and $500 \mathrm{~km} \mathrm{~s}^{-1}$ tomograms. The KMM analysis reveals the velocity distribution is trimodal with two low dispersion substructures with mean velocity $v_{\text {pec }}=$ -1620.4 and $658.8 \mathrm{~km} \mathrm{~s}^{-1}$ and dispersions $\sigma_{v_{\mathrm{pec}}}=$ 144.1 and $68.9 \mathrm{~km} \mathrm{~s}^{-1}$, respectively. The low velocity dispersions indicate that the substructures have low mass and their large peculiar velocity separation indicates they are not associated. The projection effects caused by these two separate structures aligned along the line of sight have enhanced the projected galaxy density here.

- S6: There is only a mild increase in the local galaxy surface density in this region, however the $\kappa$-test shows that the local kinematics are significantly different from the global kinematics. The KMM analysis showed that the reason for this difference was a strong bimodality with two substructures separated by a peculiar velocity of $1860 \mathrm{~km} \mathrm{~s}^{-1}$. The two substructures have mean velocity $v_{\text {pec }}=-701,1$ and $1156.5 \mathrm{~km} \mathrm{~s}^{-1}$ and dispersion $\sigma_{\text {vpec }}=522.5$ and $357.8 \mathrm{~km} \mathrm{~s}^{-1}$, respectively. The tomograms show that the negative peculiar velocity component is fairly dispersed, while the $v_{\text {pec }} \sim 1000 \mathrm{~km} \mathrm{~s}^{-1}$ component does appear to be more concentrated in this region, although it is not as prominent as other substructures such as S3. The galaxies in this region may be part of coherent substructures which are infalling from the surrounding large scale structure.

- S7: This substructure is prominent as a local overdensity in the projected galaxy distribution, although the $\kappa$-test does not reveal any significant velocity substructure here. There are local density peaks here in the $\bar{v}_{\text {pec }}=500$ and $1000 \mathrm{~km} \mathrm{~s}^{-1}$ tomograms (Figure 10) and the KMM algorithm fits a substructure with mean velocity $v_{\text {pec }}=$ $902.6 \mathrm{~km} \mathrm{~s}^{-1}$ and dispersion $\sigma_{\text {vpec }}=172.2$, although bimodality is not strongly favored. The low dispersion indicates a low mass, although given the minimal evidence for velocity substructure, the local peak in the galaxy density here may simply due to projection effects from unrelated galaxies.

\subsubsection{RXJ1720}

- S1: This substructure is a well defined local peak in the galaxy surface density distribution although it has unusual kinematic properties. The $\kappa$-test reveals significant local kinematic substructure in this region, while the tomograms reveal local overdensities in this region at $\bar{v}_{\text {pec }}=-500,-1000,500$ and $1000 \mathrm{~km} \mathrm{~s}^{-1}$ but not at $\bar{v}_{\text {pec }}=0 \mathrm{~km} \mathrm{~s}^{-1}$. The KMM analysis revealed a bimodal fit was preferred over uni- and trimodal fits with the two components having mean velocity $v_{\text {pec }}=-849.7$ and $736.0 \mathrm{~km} \mathrm{~s}^{-1}$ and dispersions of $\sigma_{\mathrm{vpec}}=594.4$ and $184.2 \mathrm{~km} \mathrm{~s}^{-1}$. The second brightest member in the core of the cluster has $v_{\text {pec }}=933 \mathrm{~km} \mathrm{~s}^{-1}$ and is within the S1 region. Okabe et al. (2010) find a second peak in their weak lensing convergence maps which is coincident with this substructure, providing further evidence that this substructure is real. We tentatively point to a mild excess in the X-ray residuals in the right panel of Figure 15 which is coincident with this structure.

- S2: This substructure has a mild enhancement in the projected galaxy distribution and a local velocity distribution which differs from the global one according to the $\kappa$-test. The KMM analysis reveals that this difference is caused by an extremely low 
velocity dispersion $\left(\sigma_{\mathrm{vpec}}=99.2 \mathrm{~km} \mathrm{~s}^{-1}\right)$ substructure at a velocity $v_{\text {pec }}=-851.0 \mathrm{~km} \mathrm{~s}^{-1}$, although the improvement in the fit in going from a unimodal to bimodal distribution is marginal. Given the small velocity dispersion and mild enhancement in galaxy surface density, this substructure is probably dynamically insignificant although we note that Okabe et al. (2010) find a weak enhancement at roughly this position in their lensing maps.

- S3: This substructure shows a mild local peak in the projected galaxy distribution and the velocity field shows a preference for positive peculiar velocities here. While the tomograms do not reveal significant local peaks for the maps centered around positive peculiar velocities, the KMM analysis reveals that the velocity distribution here is bimodal with the majority of the galaxies allocated to a component centered at $v_{\text {pec }}=818.4 \mathrm{~km} \mathrm{~s}^{-1}$ with dispersion $\sigma_{\mathrm{vpec}}=512.5$. This large dispersion, if real, indicates a significant mass in this region. However, visual inspection of the velocity distribution in the lower left panel of Figure 13 reveals that there may be some contamination from galaxies at $v_{\text {pec }} \sim 1500 \mathrm{~km} \mathrm{~s}^{-1}$ which may be inflating the dispersion. More complete spectroscopy is required in this region to better disentangle the velocity components and confirm the large dispersion measured here.

- S4: The $\kappa$-test revealed a number of large bubbles in this region which indicates evidence for velocity substructure. There is no strong galaxy surface density enhancement, although the tomogram centered at $v_{\text {pec }}=-500 \mathrm{~km} \mathrm{~s}^{-1}$ shows a local peak here while the velocity dispersion in this region appears to be low. However, the KMM analysis failed to fit a second velocity substructure to the data in this region, although the best fitting single Gaussian does have a low velocity dispersion $\left(\sigma_{\mathrm{vpec}}=577.3 \mathrm{~km} \mathrm{~s}^{-1}\right)$ and is centered at $v_{\text {pec }}=-291.1 \mathrm{~km} \mathrm{~s}^{-1}$ indicating a departure from the global velocity distribution here. More data are required in this region in order to confirm the existence of real dynamical substructure.

\section{DISCUSSION}

The principal objective of this study is to find observational evidence for merger related substructure in the sloshing cold front clusters A2142 and RXJ1720. Furthermore, we wish to determine if the cold fronts observed in the cores of these clusters can be related to merger activity, therefore confirming that cold fronts are excellent signatures of merger activity. The results of our substructure tests reveal that A2142 and RXJ1720 harbor a number of group-scale substructures which manifest themselves as enhancements in the local galaxy surface density (Section 3.2.2), and as regions of localized velocity substructure (Section 3.2.3). The existence of these group-scale substructures is consistent with a minor merger origin of the cold fronts, as seen in the simulations of Tittley \& Henriksen (2005), Ascasibar \& Markevitch (2006; see also Roediger et al. 2011 for a more specific case) where a low mass group perturbs the main cluster core and induces cold fronts. The problem now, particularly for A2142, is to determine which of the many substructures detected is the most likely perturber. As a first step, we note that substructures with large $R_{\text {proj }}$ are unlikely to have made a pericentric passage and, thus, are unlikely to be the perturber. We outline the evidence for this statement in subsection 4.1 and discuss the substructures which we consider to be the most likely perturbers in A2142 (S1 and S2; subsection 4.2.1) and RXJ1720 (S1 and S3; subsection 4.2.2).

\subsection{Ruling out the substructures least likely to be perturbers}

First, we consider the apocentric distance of a subcluster after its initial pericentric passage. We obtain an estimate for this distance by considering the simulations of Tormen et al. (2004) where the average apocentric distances for an ensemble of simulated clusters with merging subhalos of varying masses is shown in their Figure 11. We only consider results for the "self bound" satellite particles in Tormen et al. (2004), since these results are more likely to reflect our observed substructures. Two important features can be noted here: (i) On average, the subhalos do not travel farther than the main cluster's virial radius, $R_{V}$, after pericenter and (ii) Due to dynamical friction, larger mass subhalos have apocentric distances as little as $\sim 0.5 R_{V}$. Thus, it is highly unlikely that any substructure observed at radii larger than the cluster virial radius has passed pericenter - they are far more likely to be groups which are falling onto the cluster for the first time. For A2142 and RXJ1720, we assume that the virial radius is $R_{\mathrm{Vir}} \simeq R_{200}=0.17 \sigma_{v} / H(z)$ where $R_{200}$ is the radius where the density of the cluster is 200 times the critical density at redshift $z, \sigma_{v}$ is the cluster velocity dispersion and $H(z)=H_{0} \sqrt{\Omega_{m}(1+z)^{3}+\Omega_{V}}$ (Carlberg et al. 1997). This results in $R_{\mathrm{vir}}=2.36$ and $2.02 \mathrm{Mpc}$ for A2142 and RXJ1720, respectively, and we set this as an initial constraint on the $R_{\text {proj }}$ within which the perturber must lie. These limits are plotted as cyan circles in Figures 14 and 15. For RXJ1720, these limits do not strongly rule out any of the substructures as potential perturbers, although S3 lies close to the virial radius in projection. For A2142, the substructures S3 and S5 are outside of the $R_{V}$ and are therefore ruled out as potential perturbers, while S4 and S6 lie at approximately the virial radius. Those substructures at the virial radius in A2142 (S4, S6) are unlikely to be the perturbers because (i) Projection effects mean that the observed radius gives the minimum distance from the cluster center-if the substructure lies at some distance in front or behind the cluster, then it is likely to lie outside the virial radius and (ii) The limit imposed here is an upper limit on the radius. More massive systems which have passed close to the cluster core are strongly affected by dynamical friction (Tormen et al. 2004) and thus have smaller apocentric distances.

Further evidence that these substructures at large $R_{\text {proj }}\left(\gtrsim R_{V}\right)$ are unlikely to have passed pericenter comes from considering the timescales required to produce the cold front pairs seen in the Chandra images (Figures 14 and 15). Given that the fluid velocities in- 
volved are small compared to the Kepler speed (e.g., Ascasibar \& Markevitch 2006), the sloshing motions that give rise to cold fronts are likely to be reasonably well described as superpositions of internal gravity waves excited by merging subclusters (Churazov et al. 2003). Locally, the oscillation frequency of these waves is the Brunt-Väisälä frequency

$$
\omega_{\mathrm{BV}}=\sqrt{\frac{g}{r} \frac{3}{5} \frac{d \ln s}{d \ln r}}=\omega_{k} \sqrt{\frac{3}{5} \frac{d \ln s}{d \ln r}},
$$

where $g=G M(r) / r^{2}$ is the acceleration due to gravity, $r$ is the radius, $s$ is the entropy index and $\omega_{\mathrm{K}}$ is the Keplerian orbital frequency. Thus $\omega_{\mathrm{BV}}$ is expected to provide an estimate of sloshing frequencies. The radial entropy profiles for both A2142 and RXJ1720 have been measured by Cavagnolo et al. (2009) and they have logarithmic slopes of $\sim 1.22$ and 1.39 at the positions of the cold fronts, respectively. Therefore, the approximation $\omega_{\mathrm{BV}} \simeq \omega_{k}=V_{\text {circ }} / r$ is sufficient for our purposes. This justifies the estimate of Simionescu et al. (2010), which employs the radial dependence of the frequency to determine the time required for an adjacent pair of cold fronts to get out of phase by $\pi$ radians, i.e., to appear on opposite sides of the cluster center. Even in the linear approximation, a more thorough treatment requires determination of the spectrum and phasing of the internal modes excited by a merging subhalo, then following the time development of this superposition of internal gravity waves. Apart from nonlinear effects, this leaves considerable uncertainty in our time estimates.

To determine $\omega_{k}$, we measure the cold front radii from the central brightest cluster galaxy which is assumed to mark the potential minimum and assume a singular isothermal sphere (SIS) density distribution meaning the circular velocity is simply $V_{\text {circ }}=\sqrt{2} \sigma_{v}$. We also assume that the time required for the cold front pairs to become $\pi$ radians out of phase in their orbits is equivalent to the time since the perturber's pericentric passage. For A2142, the inner and outer cold fronts have radii $R_{\text {proj,in }}=87 \mathrm{kpc}$ and $R_{\text {proj,out }}=326 \mathrm{kpc}$, respectively, and $V_{\text {circ }}=1407 \mathrm{~km} \mathrm{~s}^{-1}$. The time required for the cold fronts to become out of phase by $\pi$ radians is $\tau=\pi /\left(\omega_{k, \text { in }}-\omega_{k, \text { out }}\right) \simeq 0.26$ Gyr. The same calculation for RXJ1720 with $R_{\text {proj,in }}=69 \mathrm{kpc}, R_{\text {proj,out }}=$ $185 \mathrm{kpc}$ and $V_{\text {circ }}=1247 \mathrm{~km} \mathrm{~s}^{-1}$ gives $\tau \simeq 0.27 \mathrm{Gyr}$. Roediger et al. (2011) found that the cold front age calculated by Simionescu et al. (2010) was underestimated by a factor of 3.5 when compared to their simulations. We have also calculated $\tau$ for several of the cold fronts in Figure 7 of Ascasibar \& Markevitch (2006), finding that this method generally underestimates the time since pericentric passage by factors of $3-4$, consistent with the findings of Roediger et al. . Therefore, we estimate that the "true" time since pericentric passage for both A2142 and RXJ1720 is $\tau_{\text {true }} \simeq 0.8-1.1$ Gyrs. We note that viewing angles and uncertainties in the phasing of the waves mean that these estimates give only a rough guide for the time since pericenter. Furthermore, we note that the cold fronts that we observe in A2142 and RXJ1720 occur on much larger scales than the cold fronts seen at similar times in the simulations. This may reflect differences in the physical properties of A2142 and RXJ1720 compared to the simulated clusters, e.g., the shape of the underlying potential will affect the oscillation frequencies. The spatial and temporal scales of the fronts are also likely to be affected by properties of the perturber, including its mass and impact parameter. Nevertheless, the timescales derived above provide useful estimates for our purposes and provide strong support for the radial constraints derived from Tormen et al. (2004). For a substructure to have reached the virial radius after pericenter on these timescales, it would need to have traveled at an average radial velocity since pericenter of $\sim 2100-2890$ and $1790-2460 \mathrm{~km} \mathrm{~s}^{-1}$ for A2142 and RXJ1720. While velocities of this order are expected at pericenter during a merger, they do not persist on gigayear timescales since the subcluster velocity quickly decays after pericenter due to the effects of gravity and dynamical friction as it travels outward. This places strong constraints on whether substructures S4 and S6 in A2142 have had time to have passed pericenter and traveled to their current $R_{\text {proj. }}$. Therefore, we disregard them as being potential perturbers.

Having ruled out S3, S4, S5 and S6 in A2142, we now consider substructure $\mathrm{S} 7$ which we rule out for the following reasons. First, while it lies within the virial radius, it has a relatively large radius $\left(R_{\text {proj }} \sim 1.7 \mathrm{Mpc}\right)$ which, as noted above for S4 and S6, gives its minimum distance from the cluster center. Furthermore, as noted in Section 3.3, while there is a local excess in the projected galaxy density, there is little evidence for any corresponding localized velocity substructure. This means that if there is a real substructure in this region, it is probably not very massive. A further possibility is that the excess in galaxy surface density is due to projection of galaxies which are not physically associated and therefore that S7 is not a bona fide substructure. Given its uncertain nature and large $R_{\text {proj }}$, we find it unlikely that S7 has passed pericenter and, if it has, it is probably not massive enough to have caused the perturbation required to produce the cold fronts with such large radii and contrast. Therefore, we also dismiss S7 as a potential perturber.

The radial limits derived above for RXJ1720 placed no strong limits on any of the substructures shown in Figure 15. However, we rule out the substructures S2 and S4 because they are unlikely to be significant substructures, if real associations at all. Starting with S2, this substructure consists of a grouping of a small number of galaxies with similar velocities and a small dispersion of only $\sim 100 \mathrm{~km} \mathrm{~s}^{-1}$. There appears to be a substructure at approximately this location in the lensing maps of Okabe et al. (2010), although it is not concentrated and is only detected at the $3 \sigma$ level. The presence of substructure detected using our spectroscopically confirmed members and also in the lensing maps of Okabe et al. (2010) provide strong support that S2 is a real substructure. However, the low velocity dispersion and low significance in the lensing maps imply that S2 has low mass and is therefore not likely to have played an important role in perturbing the cluster core. Considering the S4 substructure, while there are hints of localized velocity substructure in this region according to the $\kappa$-test and the velocity fields, there is only a weak excess in galaxy surface density here. Furthermore, the KMM analysis failed to identify any bimodality in this region. Given the uncertainty in the existence of velocity substructure 
and the lack of a compact 2D substructure, it is unlikely that $\mathrm{S} 4$ is a real substructure. Deeper, more complete spectroscopic coverage of RXJ1720 would be required to determine if there is a real substructure there.

\subsection{Most likely perturbers}

\subsubsection{A2142}

We begin our discussion with the S1 substructure surrounding the second BCG at $\sim 180 \mathrm{kpc}$ to the northwest of the first ranked BCG. The KMM fit returns $v_{\text {pec }}=1732.5$ and $\sigma_{v}=224.2 \mathrm{~km} \mathrm{~s}^{-1}$, although a bimodal fit to the local velocity distribution was not favored statistically over a unimodal one. However, the surrounding local overdensity in the galaxy surface density, along with the compact local overdensity in the 1500 and $2000 \mathrm{~km} \mathrm{~s}^{-1}$ tomograms (Figure 10) indicate that there is a kinematically compact grouping of $\sim 7-10$ galaxies associated with this BCG. Any kinematic distinction is likely diluted by the projection of the main cluster core galaxies onto S1 due to its close proximity in projection to the core. BCGs are expected to be found at the centers of groups and clusters thus the existence of a second $\mathrm{BCG}$ provides strong evidence that $\mathrm{S} 1$ is a merger remnant. Given the relatively high (although not extreme) positive peculiar velocity of S1, we speculate that the majority of its motion is aligned with our line of sight. In the scenario where $\mathrm{S} 1$ is the perturber responsible for the cold fronts, it must have already passed pericenter. If the estimated time since pericenter of $\sim 1$ Gyr derived above is correct, then S1 is unlikely to have passed apocenter and therefore we surmise that it is currently located on the far side of the cluster on its way to apocenter.

Further evidence for a perturber which has an orbit which is highly inclined to the plane of the sky, such as that proposed for S1, comes from the morphology of the cold fronts. The Chandra data for A2142 reveal two prominent cold fronts (Markevitch et al. 2000) straddling the cool core (Figure 14). There is no clear spiral structure seen in the residual map (right panel of Figure 14), nor in the temperature maps available in the literature (Markevitch et al. 2000, Owers et al. 2009b), although there are fairly linear structures joining the innermost cold front to the outermost one. The morphology of the cold fronts is consistent with a spiral structure viewed with its axis almost perpendicular to our line of sight, e.g., as shown in the middle panel of Figure 19 in Ascasibar \& Markevitch (2006). In the simulations, the spiral structure is formed when angular momentum is transfered to the displaced core gas from a perturber which has undergone an offcenter passage (Ascasibar \& Markevitch 2006). Because the angular momentum responsible for the spiral structure originates with the offcenter passage of the perturber, the spiral and perturber orbit must be coplanar. Based on this and the evidence that we are viewing a spiral structure with its axis almost perpendicular to our line of sight, we surmise that the perturber is orbiting in a plane which is highly inclined to the plane of the sky.

We also note here that the central BCG has a peculiar velocity of $v_{\text {pec }}=212 \pm 65 \mathrm{~km} \mathrm{~s}^{-1}$ with respect to the cluster velocity distribution. This difference is significant at the $2.9 \sigma$ level according to the method outlined in Oegerle \& Hill (2001). Assuming that the BCG lies at the bottom of the cluster potential as traced by the dark matter, we interpret this as evidence of dark matter core oscillations. As pointed out by Ascasibar \& Markevitch (2006) the observation of BCG peculiar velocities is naturally encompassed in their gasless minor merger scenario. In that scenario, a purely gravitational perturbation excites oscillations in both the dark matter and gas cores leading to cold fronts when the gas decouples from the dark matter. However, in the case where a weak shock or other purely hydrodynamical disturbance (Churazov et al. 2003, Fujita et al. 2004, Roediger et al. 2011) perturbs the core and generates the cold fronts, only the gas is expected to oscillate, thus no significant BCG peculiar velocity should be observed. We interpret the BCG peculiar velocity as evidence that the cold fronts are generated by the gravitational disturbance of a minor merger. Furthermore, the detection of a line of sight velocity offset lends support to our assertion that the perturber's orbit is inclined to the line of sight.

As an alternative, we consider the S2 substructure, which is a fairly loose group of galaxies at a projected distance of $\sim 970 \mathrm{kpc}$ from the central BCG, has a mean peculiar velocity of $v_{\text {pec }}=1680 \mathrm{~km} \mathrm{~s}^{-1}$ and dispersion $\sigma \simeq 370 \mathrm{~km} \mathrm{~s}^{-1}$. Supporting evidence for S2 being a real substructure comes from the lensing analysis of Okabe \& Umetsu (2008) who detect a signal in their projected mass map which is significant at the $3 \sigma$ level and is approximately at the position of S2. Similar to $\mathrm{S} 1$, the positive peculiar velocity of S2 implies that it is currently travelling away from us and, assuming that it has not passed apocenter, that its initial trajectory began in the foreground to the southeast of the main cluster core. It passed pericenter on the SE before being deflected behind the cluster core and to the northwest where it traveled to its current position.

\subsubsection{RXJ1720}

The most viable perturber candidate for RXJ1720 is the S1 substructure lying $\sim 550 \mathrm{kpc}$ north of the cluster core. This substructure is conspicuous as an excess in the galaxy surface density maps and shows evidence for localized kinematic substructure. A more detailed inspection of the velocity distribution surrounding $\mathrm{S} 1$ in Section 3.2 .4 revealed significant bimodality with two substructures separated by $\sim 1586 \mathrm{~km} \mathrm{~s}^{-1}$, neither of which is centered at the systemic cluster velocity $\left(\mu_{1}=-849.7 \mathrm{~km} \mathrm{~s}^{-1}\right.$ and $\left.\mu_{2}=736.0 \mathrm{~km} \mathrm{~s}^{-1}\right)$. While the velocity structure is complicated here and is hard to interpret, we are confident that there exists a massive substructure here, particularly given that Okabe et al. (2010) find significant substructure in their lensing maps which is spatially coincident with S1.

Here, we consider a scenario where S1 has perturbed the core during its pericenter passage and is currently moving outwards towards the north. Considering the Chandra residual map generated from the Chandra data in the right panel of Figure [15, a clear spiral structure can be seen which is also seen in the temperature maps of Mazzotta \& Giacintucci (2008) and Owers et al. $(2009 \mathrm{~b})$. Following on from the discussion on the spiral morphology in Section 4.2.1 this means we must be observing the spiral structure which has an axis that is aligned roughly with our line of sight. We also note that, in contrast to A2142, we see no significant peculiar ve- 
locity offset in the BCG, consistent with the sloshing occurring mainly in a plane perpendicular to our line of sight. By extension, the axis of the perturber's orbit is roughly coincident with our line of sight. Furthermore, following the spiral from the outermost cold front to the core, the winding direction is clockwise. Markevitch \& Vikhlinin (2007) noted that the orbit of the perturber and that of the gas generating the spiral pattern should exhibit prograde rotation, as seen in the simulations (Ascasibar \& Markevitch 2006, Poole et al. 2006, Roediger et al. 2011). This implies that the S1 substructure follows a clockwise orbit from our perspective. We propose that S1 has traveled from the south, passed the core region on the eastern side providing the necessary perturbation for the generation of the cold fronts and is now headed to the north. One issue with this interpretation is the proximity of $\mathrm{S} 1$ to the cluster core. If the time since pericenter of $1 \mathrm{Gyr}$ derived above is correct and the perturber's orbital plane is very close to the plane of the sky, then S1 should have had time to travel much further than $550 \mathrm{kpc}$. Of course, this assertion relies critically on the velocity of $\mathrm{S} 1$ which is not well constrained. However, even assuming a relatively small radial velocity of $1000 \mathrm{~km} \mathrm{~s}^{-1}$ since core passage implies that $\mathrm{S} 1$ should be $\sim 1 \mathrm{Mpc}$ from the cluster core. This issue is somewhat relieved if we take the lower value of $\tau_{\text {true }}=0.8$ derived above and assume that S1's orbit has some component parallel with our line of sight.

Alternatively, if we have underestimated the time since pericenter, then it is possible that S1 has passed apocenter and is currently headed towards its second pericenter. This also relieves the tension between the observed scales of the cold fronts in RXJ1720, which occur at much larger radii when compared with those formed on $\sim 1 \mathrm{Gyr}$ post-pericenter timescales seen in the simulations of Ascasibar \& Markevitch (2006). For example, in the 3.8 Gyr panel of Figure 7 in Ascasibar \& Markevitch (2006), i.e., $\sim 2.4$ Gyr after pericenter and just prior to the second pericenter, the most prominent cold fronts have radii $\sim 80 \mathrm{kpc}$ and $\sim 160 \mathrm{kpc}$, similar to the cold fronts observed in RXJ1720. However, Ascasibar \& Markevitch (2006) note that the contrast in density and temperature at the cold fronts decreases with time by a factor of 1.5 at 3.8 Gyrs, compared with the factor of $\sim 2$ jump observed in RXJ1720 (Owers et al. 2009b). This difference in contrast may reflect a difference in the mass, the gas content and/or the impact parameter of the perturber between RXJ1720 and the simulations presented in Figure 7 of Ascasibar \& Markevitch.

The S3 substructure located at $\sim 1.7 \mathrm{Mpc}$ south of the cluster core may also be considered a potential perturber. The KMM fits revealed that the velocity of this substructure is $\sim 820 \mathrm{~km} \mathrm{~s}^{-1}$ and it has dispersion $\sigma \sim 512 \mathrm{~km} \mathrm{~s}^{-1}$. This dispersion is fairly large and comparable to that expected of a reasonably large group or poor cluster. However, visual inspection of the velocity distribution (lower left panel in Figure 13) reveals that the $820 \mathrm{~km} \mathrm{~s}^{-1}$ partition has two peaks and thus that this partition may be contaminated by galaxies which are not associated with the substructure. This contamination may be responsible for inflating the velocity dispersion. More data are needed to disentangle any contamination and to confirm this velocity dispersion. In any case, there is clearly a dynamical substructure in this region. In the scenario where S3 is the perturber, we propose that it has traveled from the north and is currently heading to the south. Based on the arguments above on the winding direction of the spiral structure seen in the right panel of Figure 15, we suggest that S3 passed the cluster core on the western side and is currently heading towards apocenter for the first time.

\section{SUMMARY AND CONCLUSIONS}

We have presented comprehensive MMT/Hectospec and Keck/DEIMOS multi-object spectroscopy of the cold front clusters A2142 and RXJ1720. These clusters were selected from the "relaxed appearing" subsample of cold front clusters from Owers et al. (2009b) with the specific aim of testing the hypothesis that minor mergers cause the observed cold fronts. To do this, we used the spatial and kinematic information contained in samples of 956 and 400 spectroscopically confirmed cluster members for A2142 and RXJ1720, respectively, to search for substructure. In both of the clusters we find group-sized substructure and we identify likely perturbers based on comparisons between the observed cold front morphologies and those seen in the simulations. Our results are consistent with the cold fronts being caused by sloshing induced by the gravitational perturbation caused by a minor merger. We stress, however, that more detailed merger histories and better orbital constraints will likely require cluster-specific simulations aimed at reproducing both the X-ray morphology and the positions and kinematic properties of the detected substructures. Nonetheless, we conclude that our original stated goal of showing cold fronts are related to merger activity (Owers et al. $2009 \mathrm{c}, \mathrm{a}, \mathrm{b}$ ) has been met.

We thank Michael Cooper and Jeffrey Newman for their help with spec2d. MSO and WJC gratefully acknowledge the financial support of an Australian Research Council Discovery Project grant throughout the course of this work. PEJN was partly supported by NASA grant NAS8-03060. We acknowledge the Keck data used in this paper was obtained through the Swinburne Time Allocation Committee for Keck (STACK). The authors wish to recognize and acknowledge the very significant cultural role and reverence that the summit of Mauna Kea has always had within the indigenous Hawaiian community. We are most fortunate to have the opportunity to conduct observations from this mountain. Some of the observations reported here were obtained at the MMT Observatory, a joint facility of the Smithsonian Institution and the University of Arizona. We thank the MMT operators and queue-schedule mode scientists for their help during observations and the staff at the Harvard-Smithsonian Center for Astrophysics Telescope Data Center for reducing the Hectospec data.

Facilities: CXO (ACIS), Keck (DEIMOS), MMT (Hectospec)

\section{REFERENCES}

Ascasibar, Y., \& Markevitch, M. 2006, ApJ, 650, 102 Ashman, K. M., Bird, C. M., \& Zepf, S. E. 1994, AJ, 108, 2348 Beers, T. C., Flynn, K., \& Gebhardt, K. 1990, AJ, 100, 32 Biviano, A., \& Katgert, P. 2004, A\&A, 424, 779 

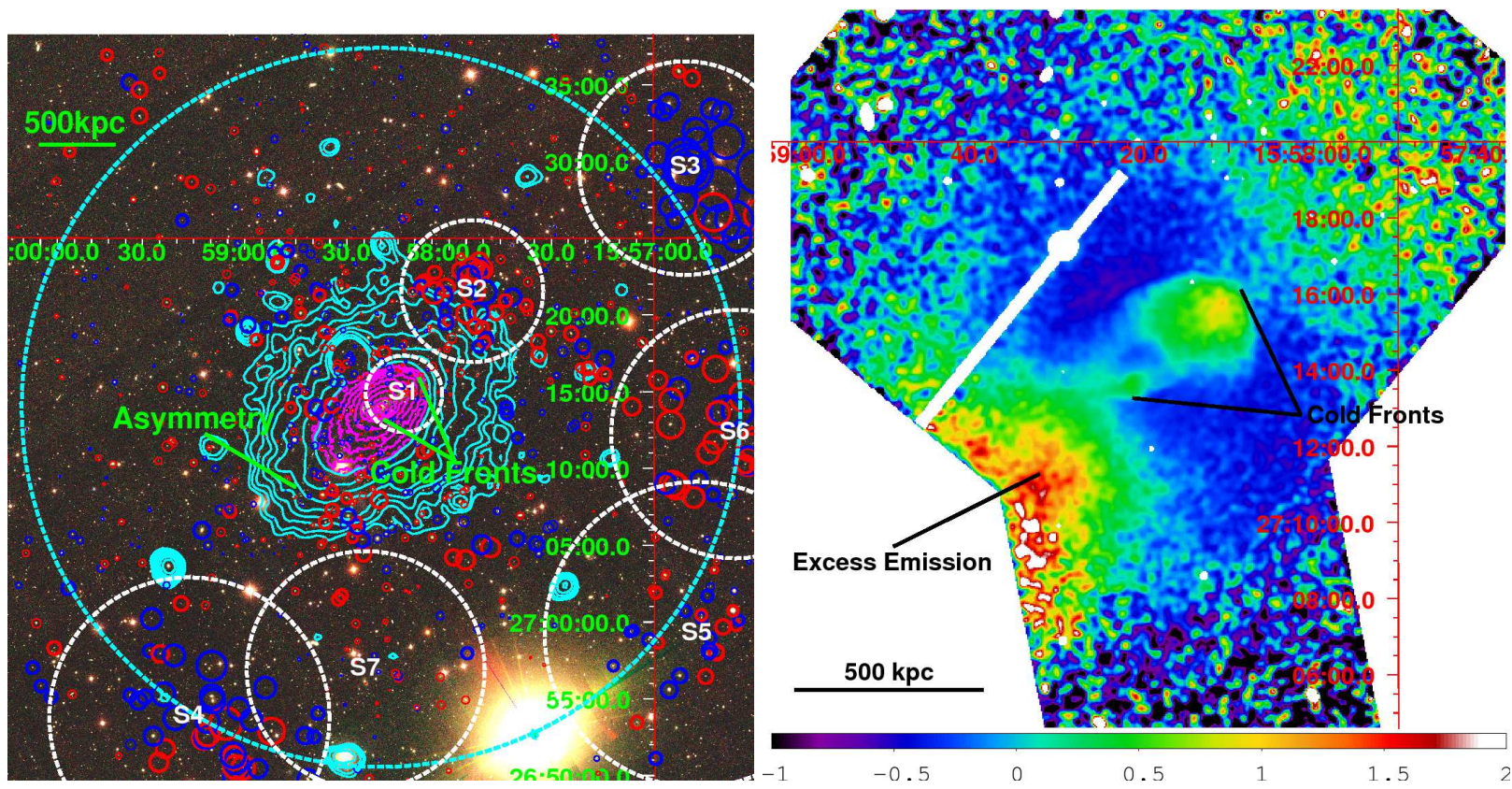

Figure 14. Left panel: SDSS gri image of A2142 with X-ray contours from ROSAT (cyan) and Chandra (magenta) overlaid. The white dashed circles show the same regions used to define the KMM substructures in Figure 12 The cyan dashed circle shows the cluster virial radius $\left(R_{V}=2.36 \mathrm{Mpc}\right)$ beyond which we do not expect to find substructure which has passed pericenter. Right panel: Residuals from an exposure and background corrected $0.5-7 \mathrm{keV}$ Chandra image after subtraction of the azimuthal average at each radius. There is no clear spiral structure joining the outer cold front to the cluster core in this residual map.
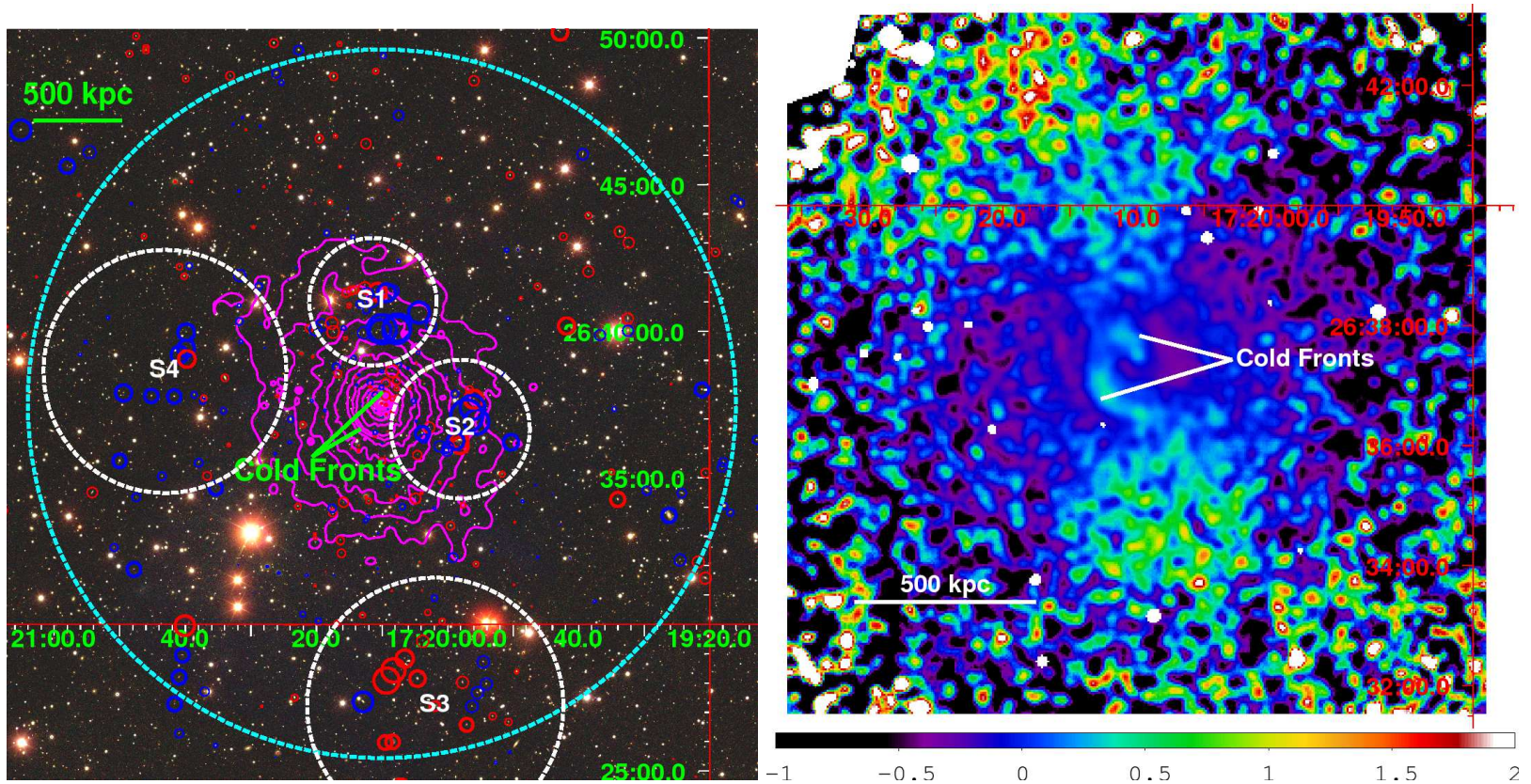

Figure 15. Left and right panels: The same as Figure 14 but for RXJ1720. ROSAT contours are not shown in the left panel, and the radius of the cyan dashed circles is $R_{V}=2.02 \mathrm{Mpc}$.

Biviano, A., Katgert, P., Mazure, A., Moles, M., den Hartog, R., Perea, J., \& Focardi, P. 1997, A\&A, 321, 84

Buote, D. A., \& Tsai, J. C. 1996, ApJ, 458, 27

Cappellari, M., \& Copin, Y. 2003, MNRAS, 342, 345

Carlberg, R. G., Yee, H. K. C., \& Ellingson, E. 1997, ApJ, 478, 462

Cavagnolo, K. W., Donahue, M., Voit, G. M., \& Sun, M. 2009, ApJS, 182, 12

Churazov, E., Forman, W., Jones, C., \& Böhringer, H. 2003, ApJ, 590,225

Colless, M., \& Dunn, A. M. 1996, ApJ, 458, 435
Cross, N. J. G., Driver, S. P., Liske, J., Lemon, D. J., Peacock, J. A., Cole, S., Norberg, P., \& Sutherland, W. J. 2004, MNRAS, 349, 576

David, L. P., \& Kempner, J. 2004, ApJ, 613, 831 
Davis, M., Faber, S. M., Newman, J., Phillips, A. C., Ellis, R. S., Steidel, C. C., Conselice, C., Coil, A. L., Finkbeiner, D. P., Koo, D. C., Guhathakurta, P., Weiner, B., Schiavon, R., Willmer, C., Kaiser, N., Luppino, G. A., Wirth, G., Connolly, A., Eisenhardt, P., Cooper, M., \& Gerke, B. 2003, in Presented at the Society of Photo-Optical Instrumentation Engineers (SPIE) Conference, Vol. 4834, Society of Photo-Optical Instrumentation Engineers (SPIE) Conference Series, ed. P. Guhathakurta, 161-172

den Hartog, R., \& Katgert, P. 1996, MNRAS, 279, 349

Diehl, S., \& Statler, T. S. 2006, MNRAS, 368, 497

Dressler, A., \& Shectman, S. A. 1988, AJ, 95, 985

Elvis, M., Plummer, D., Schachter, J., \& Fabbiano, G. 1992, ApJS, 80, 257

Fabricant, D., Fata, R., Roll, J., Hertz, E., Caldwell, N., Gauron, T., Geary, J., McLeod, B., Szentgyorgyi, A., Zajac, J., Kurtz, M., Barberis, J., Bergner, H., Brown, W., Conroy, M., Eng, R. Geller, M., Goddard, R., Honsa, M., Mueller, M., Mink, D., Ordway, M., Tokarz, S., Woods, D., Wyatt, W., Epps, H., \& Dell'Antonio, I. 2005, PASP, 117, 1411

Fujita, Y., Matsumoto, T., \& Wada, K. 2004, ApJ, 612, L9

Ghizzardi, S., Rossetti, M., \& Molendi, S. 2010, A\&A, 516, A32+

Gutierrez, K., \& Krawczynski, H. 2005, ApJ, 619, 161

Hallman, E. J., \& Markevitch, M. 2004, ApJ, 610, L81

Henry, J. P., \& Briel, U. G. 1996, ApJ, 472, 137

Hlavacek-Larrondo, J., Fabian, A. C., Sanders, J. S., \& Taylor, G. B. 2011, MNRAS, 905

Horne, K. 1986, PASP, 98, 609

Kempner, J. C., \& David, L. P. 2004, MNRAS, 349, 385

Kurtz, M. J., Mink, D. J., Wyatt, W. F., Fabricant, D. G., Torres, G., Kriss, G. A., \& Tonry, J. L. 1992, in Astronomical Society of the Pacific Conference Series, Vol. 25, Astronomical Data Analysis Software and Systems I, ed. D. M. Worrall, C. Biemesderfer, \& J. Barnes, 432-+

López-Cruz, O., Barkhouse, W. A., \& Yee, H. K. C. 2004, ApJ, 614,679

Markevitch, M., Gonzalez, A. H., David, L., Vikhlinin, A., Murray, S., Forman, W., Jones, C., \& Tucker, W. 2002, ApJ, $567, \mathrm{~L} 27$

Markevitch, M., Ponman, T. J., Nulsen, P. E. J., Bautz, M. W., Burke, D. J., David, L. P., Davis, D., Donnelly, R. H., Forman, W. R., Jones, C., Kaastra, J., Kellogg, E., Kim, D.-W., Kolodziejczak, J., Mazzotta, P., Pagliaro, A., Patel, S., Van Speybroeck, L., Vikhlinin, A., Vrtilek, J., Wise, M., \& Zhao, P. 2000, ApJ, 541, 542

Markevitch, M., \& Vikhlinin, A. 2007, Phys. Rep., 443, 1

Markevitch, M., Vikhlinin, A., \& Mazzotta, P. 2001, ApJ, 562, L153

Mazzotta, P., \& Giacintucci, S. 2008, ApJ, 675, L9

Mazzotta, P., Markevitch, M., Vikhlinin, A., Forman, W. R., David, L. P., \& VanSpeybroeck, L. 2001, ApJ, 555, 205

Mink, D. J., Wyatt, W. F., Caldwell, N., Conroy, M. A., Furesz, G., \& Tokarz, S. P. 2007, in Astronomical Society of the Pacific Conference Series, Vol. 376, Astronomical Data Analysis Software and Systems XVI, ed. R. A. Shaw, F. Hill, \& D. J. Bell, 249-+
Miller, C. J., Nichol, R. C., Reichart, D., Wechsler, R. H., Evrard, A. E., Annis, J., McKay, T. A., Bahcall, N. A., Bernardi, M., Boehringer, H., Connolly, A. J., Goto, T., Kniazev, A., Lamb, D., Postman, M., 2005, AJ, 130, 968

Oegerle, W. R., \& Hill, J. M. 2001, AJ, 122, 2858

Oegerle, W. R., Hill, J. M., \& Fitchett, M. J. 1995, AJ, 110, 32

Okabe, N., Takada, M., Umetsu, K., Futamase, T., \& Smith, G. P. 2010, PASJ, 62,811

Okabe, N., \& Umetsu, K. 2008, PASJ, 60, 345

Owers, M. S., Couch, W. J., \& Nulsen, P. E. J. 2009a, ApJ, 693, 901

Owers, M. S., Nulsen, P. E. J., Couch, W. J., \& Markevitch, M. 2009b, ApJ, 704, 1349

Owers, M. S., Nulsen, P. E. J., Couch, W. J., Markevitch, M., \& Poole, G. B. 2009c, ApJ, 692, 702

Owers, M. S., Randall, S. W., Nulsen, P. E. J., Couch, W. J., David, L. P., \& Kempner, J. C. 2011, ApJ, 728, 27

Pinkney, J., Roettiger, K., Burns, J. O., \& Bird, C. M. 1996, ApJS, 104, 1

Poole, G. B., Fardal, M. A., Babul, A., McCarthy, I. G., Quinn, T., \& Wadsley, J. 2006, MNRAS, 373, 881

Roediger, E., Brüggen, M., Simionescu, A., Böringer, H., Churazov, E., \& Forman, W. R. 2011, MNRAS, 369

Russell, H. R., Sanders, J. S., Fabian, A. C., Baum, S. A., Donahue, M., Edge, A. C., McNamara, B. R., \& O'Dea, C. P. 2010, MNRAS, 406, 1721

Simionescu, A., Werner, N., Forman, W. R., Miller, E. D., Takei, Y., Böhringer, H., Churazov, E., \& Nulsen, P. E. J. 2010, MNRAS, 405, 91

Stoughton, C., Lupton, R. H., Bernardi, M., Blanton, M. R., \& et al. 2002, AJ, 123,485

Tittley, E. R., \& Henriksen, M. 2005, ApJ, 618, 227

Tormen, G., Moscardini, L., \& Yoshida, N. 2004, MNRAS, 350, 1397

Umetsu, K., Birkinshaw, M., Liu, G.-C., Wu, J.-H. P., Medezinski, E., Broadhurst, T., Lemze, D., Zitrin, A., Ho, P. T. P., Huang, C.-W. L., Koch, P. M., Liao, Y.-W., Lin, K.-Y., Molnar, S. M., Nishioka, H., Wang, F.-C., Altamirano, P., Chang, C.-H., Chang, S.-H., Chang, S.-W., Chen, M.-T., Han, C.-C., Huang, Y.-D., Hwang, Y.-J., Jiang, H., Kesteven, M., Kubo, D. Y., Li, C.-T., Martin-Cocher, P., Oshiro, P., Raffin, P., Wei, T., \& Wilson, W. 2009, ApJ, 694, 1643

Vikhlinin, A., Markevitch, M., \& Murray, S. S. 2001, ApJ, 551, 160

Wojtak, R., \& Lokas, E. L. 2007, MNRAS, 377, 843

Wojtak, R., Łokas, E. L., Mamon, G. A., Gottlöber, S., Prada, F., \& Moles, M. 2007, A\&A, 466, 437

Zabludoff, A. I., Franx, M., \& Geller, M. J. 1993, ApJ, 419, 47 